\title{
SLICE CONVERGENCE : STABILITÉ ET OPTIMISATION DANS LES ESPACES NON RÉFLEXIFS
}

\author{
Khalid El Hajioui ${ }^{1}$ et Driss Mentagui ${ }^{1}$
}

\begin{abstract}
Résumé. Il est démontré par Mentagui [ESAIM: COCV 9 (2003) 297-315] que, dans le cas des espaces de Banach généraux, la convergence d'Attouch-Wets est stable par une classe d'opérations classiques de l'analyse convexe, lorsque les limites des suites d'ensembles et de fonctions satisfont certaines conditions de qualification naturelles. Ceci tombe en défaut avec la slice convergence. Dans cet article, nous établissons des conditions de qualification uniformes assurant la stabilité de la slice convergence et de la slice convergence duale par les mêmes opérations, dont le rôle est fondamental en optimisation convexe. Nous obtenons comme conséquences certains résultats clés de stabilité de l'épi-convergence établis par Mc Linden et Bergstrom [Trans. Amer. Math. Soc. 286 (1981) 127-142] en dimension finie. Comme application, nous présentons un modèle de convergence et de stabilité recouvrant une large classe de problèmes en optimisation convexe et en théorie de la dualité. Les éléments clés dans notre démarche sont l'analyse d'horizon, les notions de quasi-continuité et d'inflocale compacité des fonctions convexes, puis la bicontinuité de la transformation de Legendre-Fenchel relativement à la slice convergence et la slice convergence duale.
\end{abstract}

\begin{abstract}
It is shown by Mentagui [ESAIM COCV 9 (2003) 297-315] that, in the case of general Banach spaces, the Attouch-Wets convergence is stable by a class of classical operations of convex analysis, when the limits satisfy some natural qualification conditions. This fails with the slice convergence. We establish here uniform qualification conditions ensuring the stability of the slice convergence under the same operations which play a basic role in convex optimization. We obtain as consequences, some key stability results of epi-convergence established by Mc Linden and Bergstrom [Trans. Amer. Math. Soc. 286 (1981) 127-142] in finite dimension. As an application, we give a model of convergence and stability for a wide class of problems in convex optimization and duality theory. The key ingredients in our methodology are, the horizon analysis, the notions of quasi-continuity and inf-local compactness of convex functions, and the bicontinuity of the Legendre-Fenchel transform relatively to the slice convergence.
\end{abstract}

Classification Mathématique. 90C25, 90C31, 49K40, 46N10.

Reçu le 22 janvier 2003.

\footnotetext{
Mots Clés. Fonction convexe, opérateur linéaire, slice convergence, Mosco-convergence, épi-convergence, convergence uniforme sur les bornés, inf-locale compacité, quasi-continuité, cône (fonction) horizon, dualité, stabilité, optimisation convexe.

1 Laboratoire d'Analyse Convexe et Variationnelle, Systèmes Dynamiques et Processus Stochastiques, Département de Mathématiques, Faculté des Sciences, Université Ibn Tofail, BP 133, Kénitra, Maroc ;

e-mail : elhajkhalid@hotmail.com; d_mentagui@hotmail.com
} 


\section{INTRODUCTION}

L'étude de la stabilité des problèmes variationnels en analyse non linéaire est considérée comme un des thèmes les plus importants de la littérature dans ce domaine [5, 6, 8, 24, 25, 50-53]. L'objet général de cette étude est l'analyse de l'influence « des petites perturbations » de certains paramètres sur les solutions du problème initial. En théorie d'approximation, ce problème peut être abordé de la manière suivante : sachant qu'on connaisse une suite de problèmes approchant dans un certain sens un problème variationnel limite éventuellement inconnu, sous-quelles conditions les solutions de ces problèmes (quand elles existent) convergent vers une solution du problème limite ? L'étude de ce type de problèmes a connu un développement considérable depuis que Hadamard a introduit la notion des problèmes bien posés en analyse non linéaire pour étudier la stabilité d'une classe de problèmes de la physique mathématique [24,25]. Ensuite, Tikhonov a apporté dès 1963 un apport fort intéressant pour stabiliser une large classe de problèmes mal posés en physique mathématique et en théorie du contrôle optimal [44-47]. Avec l'introduction des convergences variationnelles par Wijsman, suite à ses travaux en théorie de la décision statistique $[49,50]$, puis par Mosco dans le cadre de l'approximation des solutions d'une classe d'inéquations variationnelles [37,38], ce thème fût intensivement étudié sous différentes formes et a trouvé ses principales applications en optimisation paramétrique et en programmation mathématique [22, 42, 51,52], en théorie d'approximation et celle du contrôle optimal $[1,12,13,20]$, puis celle des problèmes d'optimisation bien posés et du calcul des variations $[20,32,42]$. Aussi, le développement considérable des études sur les convergences variationnelles qui constituent un outil puissant et efficace pour l'étude de la stabilité et l'approximation d'une large classe de problèmes variationnels, a permis de dégager plusieurs importantes notions de convergences, dont l'une est la slice convergence qui est une extension naturelle de la convergence au sens de Mosco dans le cas non réflexif. Cette convergence a été introduite formellement pour la première fois dans [43] puis étudiée intensivement par divers auteurs $[3,9,10,14,22,23,35]$. Elle est en général plus fine que la convergence au sens de Mosco et coïncide avec cette dernière si et seulement si l'espace considéré est réflexif [11]. De plus, elle conserve la bicontinuité de la transformation de Legendre-Fenchel $: f \in \Gamma(X) \longrightarrow f^{*} \in \Gamma\left(X^{*}\right)$ dans le cas d'un espace normé quelconque [10]. Cette propriété fondamentale justifie le rôle primordial que peut jouer cette convergence en analyse non linéaire, notamment en optimisation convexe et en théorie de la dualité dans les espaces normés non réflexifs.

L'objet de cet article est de montrer que, sous certaines conditions de qualification uniformes, la slice convergence est stable par certaines opérations classiques de l'analyse convexe dont le rôle est fondamental en optimisation convexe et en théorie de l'approximation. L'hypothèse clé, dans ces conditions de qualification, est la notion d'inf-locale compacité d'une fonction de $\Gamma(X)$ qui se traduit dans l'espace dual par la quasi-continuité de sa transformée de Legendre-Fenchel [26]. Nous retrouvons ainsi certains résultats de stabilité de Mc Linden et Bergstrom [30] en dimension finie. Notre étude s'achève enfin par une application à la théorie de la dualité en optimisation convexe dans un cadre non nécessairement réflexif.

\section{PrÉliminaires}

Soit $X$ un espace de Banach d'origine $\theta$ et de boule unité fermée $U$. Son dual topologique noté $X^{*}$ est d'origine $\theta^{*}$ et de boule unité fermée $U^{*}$. $\|\cdot\|$ et $\|\cdot\|_{*}$ désignent respectivement les normes de $X$ et $X^{*}$, $w$ et $w^{*}$ les topologies faibles $\sigma\left(X, X^{*}\right)$ et $\sigma\left(X^{*}, X\right)$, et $\tau^{*}$ la topologie de Mackey $\tau\left(X^{*}, X\right)$. $\pi\left(\right.$ resp. $\left.\pi^{*}\right)$ désignera dans la suite une topologie d'espace localement convexe séparé (e.l.c.s.) compatible avec la dualité $\left\langle X, X^{*}\right\rangle$ (resp. $\left.\left\langle X^{*}, X\right\rangle\right)$.

Sans aucune ambiguité, les notations précédentes resteront les mêmes pour tout autre espace de Banach $Y$ de dual topologique $Y^{*}$.

Si $f \in \overline{\mathbb{R}}^{X}$, on note le domaine effectif de $f$ et son épigraphe par :

$$
\text { Dom } f:=\{x \in X / f(x)<+\infty\} \text {, epi } f:=\{(x, \alpha) \in X \times \mathbb{R} / f(x) \leq \alpha\}
$$


La transformée de Legendre-Fenchel de $f$ notée $f^{*}$ est la fonction définie pour tout $y \in X^{*}$ par $f^{*}(y):=$ $\sup \{\langle x, y\rangle-f(x) / x \in D o m f\}$. On dit que $f$ est propre si Dom $f \neq \emptyset$ et $f(x)>-\infty$ pour tout $x \in X$. $\Gamma(X)$ (resp. $\left.\Gamma\left(X^{*}\right)\right)$ désigne l'ensemble des fonctions propres convexes semi-continues inférieurement (sci) (resp. $w^{*}$-sci). $\mathcal{C}(X)$ (resp. $\mathcal{C}\left(X^{*}\right)$ ) désigne l'ensemble des parties non vides convexes fermées de $X$ (resp. non vides convexes $w^{*}$-fermées de $\left.X^{*}\right)$.

Pour $f, g \in \Gamma(X)$ et $C \in \mathcal{C}(X)$ on rappelle les définitions suivantes : - l'inf-convolution de $f$ et $g$ est la fonction notée $f \# g$ définie pour tout $x \in X \operatorname{par}(f \# g)(x):=\inf \{f(u)+g(x-u) / u \in X\}$.

Pour l'intêret de cette opération on pourra consulter $[4,29,33,36]$;

- la fonction indicatrice de $C$ est la fonction notée $I_{C}$ avec

$$
I_{C}(x):=\left\{\begin{array}{c}
0 \text { si } x \in C \\
+\infty \text { si } x \notin C
\end{array}\right.
$$

- la jauge de $C$ est la fonction notée $j_{C}$ (ou tout simplement $j$ lorsque aucune ambiguité n'est à craindre) et définie pour tout $x \in X$ par $j_{C}(x):=\inf \{\alpha>0 / x \in \alpha C\}$ avec la convention inf $\emptyset=+\infty$.

On vérifie aisément que si $C$ est un voisinage de $\theta$ convexe fermé équilibré pour une topologie d'e.l.c.s. définie sur $X$, alors $j_{C}$ est une semi-norme continue et $C=\left\{x \in X / j_{C}(x) \leq 1\right\}$ [39];

- le sous-différentiel de $f$ en $x$ au sens de l'analyse convexe est donné par

$$
\partial f(x):=\left\{y \in X^{*} / f(x)+f^{*}(y)=\langle x, y\rangle\right\},
$$

et le graphe de la multifonction $x \in X \longrightarrow \partial f(x) \subset X^{*}$ est noté par

$$
G(\partial f):=\left\{(x, y) \in X \times X^{*} / y \in \partial f(x)\right\} ;
$$

- le cône horizon (ou asymptote) de $C$ est l'ensemble noté $C^{\infty}$ avec

$$
C^{\infty}:=\cap_{\varepsilon>0} \varepsilon\left(C-x_{0}\right) \text {, où } x_{0} \in C ;
$$

- la fonction horizon (ou asymptote) de $f$ est la fonction notée $f^{\infty}$ et définie pour tout $x \in X$ par

$$
f^{\infty}(x):=\sup _{\lambda>0} \frac{f\left(x_{0}+\lambda x\right)-f\left(x_{0}\right)}{\lambda} \text {, où } x_{0} \in \operatorname{Dom} f .
$$

Il est connu $[29,41,42]$ que $C^{\infty}$ (resp. $f^{\infty}$ ) ne dépend pas du choix de $x_{0}$ dans $C$ (resp. Dom $f$ ) et que

$$
\text { epi } f^{\infty}=(\text { epif })^{\infty} \quad \text { et } f^{* \infty}(y)=\sup \{\langle x, y\rangle / x \in \operatorname{Dom} f\}, \forall y \in X^{*} \text {; }
$$

- $f$ est dite quasi-continue [26] si son domaine effectif engendre une variété affine fermée $L_{(f)}$ de codimension finie et si la restriction de $f$ à $L_{(f)}$ est continue en tout point de l'intérieur de $D o m f$ relativement à $L_{(f)}$, noté $\operatorname{ir}(\operatorname{Dom} f)$. $C$ est dit quasi-continu si $I_{C}$ est quasi-continue.

Il est clair que si $f$ est convexe et continue en un point de $\operatorname{Dom} f$, alors $f$ est quasi-continue; et si $X$ est de dimension finie alors toute fonction de $\Gamma(X)$ est quasi-continue;

- on dit que $f$ et $g$ forment un couple uni si leurs domaines effectifs ne sont que trivialement séparés, c'est-à-dire si un hyperplan fermé sépare les deux domaines, il les contient tous les deux. On dira que deux convexes de $X$ sont unis si leurs fonctions indicatrices forment un couple uni.

En dimension finie, deux convexes $A$ et $B$ sont unis si et seulement si $\operatorname{ir}(A) \cap \operatorname{ir}(B) \neq \emptyset[41]$.

Rappelons ici le lemme suivant qui nous sera utile dans la suite :

Lemme 2.1 [34]. Soient $X$ un espace de Banach et $f, g$ deux fonctions de $\Gamma(X)$ avec $f$ quasi-continue. Les propriétés suivantes sont équivalentes : 
1) $f$ et $g$ forment un couple uni ;

2) $E:=\left(\text { epi } f^{*}\right)^{\infty} \cap-\left(\text { epi } g^{*}\right)^{\infty}$ est un s.e.v. $\operatorname{de} X^{*} \times \mathbb{R}$;

3) $F:=\left\{x^{*} \in X^{*} / f^{* \infty}\left(x^{*}\right)+g^{* \infty}\left(-x^{*}\right) \leq 0\right\}$ est un s.e.v. de $X^{*}$;

4) $\bar{M}$ est un s.e.v. de $X$ avec $M:=\cup_{\lambda \geq 0} \lambda(\operatorname{Dom} f-D o m g)$;

5) $M$ est un s.e.v. fermé de $X$.

Si une de ces conditions est satisfaite, alors $\operatorname{proj}_{X^{*}} E=F=M^{\perp}$.

Si maintenant $E$ est un sous-ensemble non vide de $X$. On note,

$$
E^{-}:=\{A \in \mathcal{C}(X) / A \cap E \neq \emptyset\}, E^{++}:=\{A \in \mathcal{C}(X) / \exists \varepsilon>0: A+\varepsilon U \subset E\} .
$$

Définition 2.2 [10]. Soit $X$ un espace de Banach. La slice topologie $\tau_{s}$ définie $\operatorname{sur} \mathcal{C}(X)$ est la topologie qui a pour sous-base tous les ensembles de la forme $V^{-}$où $V$ est un $\|\cdot\|$-ouvert de $X$, plus ceux de la forme $\left(B^{c}\right)^{++}$ où $B$ est une partie non vide convexe $\|\cdot\|$-fermée bornée de $X$.

On définit de même la slice topologie duale $\tau_{s}^{*}$ sur $\mathcal{C}\left(X^{*}\right)$ comme étant la topologie qui a pour sous-base tous les ensembles de la forme $V^{-}$où $V$ est un $\|\cdot\|_{*}$-ouvert de $X^{*}$ et ceux de la forme $\left(K^{c}\right)^{++}$où $K$ est un sous-ensemble non vide convexe $w^{*}$-compact quelconque de $X^{*}$.

Il est prouvé dans $[9,10,14]$ que la slice topologie définie sur $\mathcal{C}(X)$ est la topologie la moins fine rendant continues toutes les fonctions « gap $»(D(B, .))_{B}$ avec

$$
D(B, .): A \in \mathcal{C}(X) \longrightarrow D(B, A):=\inf \{\|b-a\| /(a, b) \in A \times B\} \in \mathbb{R}^{+}
$$

où $B$ est une partie non vide convexe $\|$.\|-fermée bornée quelconque de $X$. Si on remplace dans la définition précédente « $B$ convexe fermé borné » par « $B$ faiblement compact » on retrouve la topologie de Mosco $[1,11$, $26,38]$.

Soient maintenant $f_{n}, n \in \mathbb{N}, f$ des fonctions de $\Gamma(X)$ et $C_{n}, n \in \mathbb{N}, C$ des éléments de $\mathcal{C}(X)$ :

(1) on dit que la suite $\left(f_{n}\right)_{n}$ épi-converge vers $f$ (pour la topologie de la norme) et on note $f_{n} \stackrel{e}{\longrightarrow} f$ si les conditions suivantes sont satisfaites [1] :

(i) $\forall x \in X, \forall x_{n} \stackrel{\|\cdot\|}{\longrightarrow} x, f(x) \leq \underline{\lim } f_{n}\left(x_{n}\right)$;

(ii) $\forall x \in X, \exists x_{n} \stackrel{\|\cdot\| \|}{\longrightarrow} x, f_{n}\left(x_{n}\right) \longrightarrow f(x)$.

$\operatorname{Si}\left(f_{n}\right)_{n}$ épi-converge vers $f$ à la fois pour la topologie forte et la topologie faible de $X,\left(f_{n}\right)_{n}$ est dite alors convergente au sens de Mosco vers $f$ et on note $f_{n} \stackrel{M}{\longrightarrow} f[1,26]$;

(2) on dit que $\left(f_{n}\right)_{n}$ est slice convergente vers $f$ et on note $f_{n} \stackrel{\tau_{s}}{\longrightarrow} f$ si et seulement si la suite des épigraphes $\left(\text { epi } f_{n}\right)_{n}$ converge vers l'épigraphe epi $f$ pour la slice topologie définie sur $\mathcal{C}(X \times \mathbb{R})$ conformément à la définition 2.2. Ce qui est encore équivalent $[3,10]$ à l'une des deux conditions (a) et (b) suivantes :
(a) $\left\{\begin{array}{c}(i) \forall x \in X, \exists x_{n} \stackrel{\|\cdot\|}{\longrightarrow} x \text { telle que } f_{n}\left(x_{n}\right) \longrightarrow f(x) ; \\ \left(\text { ii } \forall \forall\left(x_{n}\right)_{n} \text { une suite bornée } \subset X, \forall(y, \eta) \text { tel que }\right. \\ \eta>f^{*}(y), f_{n}\left(x_{n}\right)>\left\langle y, x_{n}\right\rangle-\eta \text { pour } n \text { assez grand. }\end{array}\right.$
(b) $\left\{\begin{array}{c}(i) \forall x \in X, \exists x_{n} \stackrel{\|\cdot\| \cdot \|}{\|\cdot\|_{*}} x \text { telle que } f_{n}\left(x_{n}\right) \longrightarrow f(x) ; \\ \text { (ii) } \forall y \in X^{*}, \exists y_{n} \stackrel{\longrightarrow}{\longrightarrow} y \text { telle que } f_{n}^{*}\left(y_{n}\right) \longrightarrow f^{*}(y) \text {. }\end{array}\right.$

Il est important de noter à ce niveau que la transformation de Young-Fenchel $f \longrightarrow f^{*}$ est un homéomorphisme de $\left(\Gamma(X), \tau_{s}\right)$ sur $\left(\Gamma\left(X^{*}\right), \tau_{s}^{*}\right)$ pour tout espace vectoriel normé (e.v.n.) [10]. De plus, il est connu que la slice convergence est strictement plus fine que la convergence au sens de Mosco et que les deux convergences coïncident si et seulement si $X$ est un espace de Banach réflexif [10,11];

(3) on dit que $\left(C_{n}\right)_{n}$ converge au sens de Painlevé-Kuratowski vers $C$ et on note $C_{n} \stackrel{P . K}{\longrightarrow} C$ si et seulement si $I_{C_{n}} \stackrel{e}{\longrightarrow} I_{C}$, ou de façon équivalente :
(i) $\forall x \in C, \quad \exists x_{n} \in C_{n}$ telle que $x_{n} \stackrel{\|.\|}{\longrightarrow} x$;
(ii) $\forall\left(n_{k}\right)_{k}$ une sous-suite, si $x_{k} \in C_{n_{k}}$ et $x_{k} \stackrel{\|\cdot\|}{\longrightarrow} x$ alors $x \in C$. 
De même, on dit que $\left(C_{n}\right)_{n}$ converge au sens de Mosco (resp. slice convergente) vers $C$ et on note $C_{n} \stackrel{M}{\longrightarrow} C$ (resp. $C_{n} \stackrel{\tau_{s}}{\longrightarrow} C$ ) si et seulement si $I_{C_{n}} \stackrel{M}{\longrightarrow} I_{C}$ (resp. $\left.I_{C_{n}} \stackrel{\tau_{s}}{\longrightarrow} I_{C}\right)[1,10,38]$.

Soient $Y$ un autre espace de Banach et $A_{n}, n \in \mathbb{N}, A: X \longrightarrow Y$ des opérateurs linéaires continus. On dit que la suite $\left(A_{n}\right)_{n}$ converge simplement vers $A$ et on note $A_{n} \longrightarrow A$ si $\left\|A_{n}(x)-A(x)\right\| \longrightarrow 0 \forall x \in X$; et que $\left(A_{n}\right)_{n}$ converge vers $A$ uniformément sur les bornés de $X$ et on note $A_{n} \stackrel{U B}{\longrightarrow} A$ si $\sup _{\|x\| \leq \rho}\left\|A_{n}(x)-A(x)\right\| \longrightarrow 0$ $\forall \rho>0$.

\section{VERSION ENSEMBLISTE DE LA STABILITÉ}

Dans cette section, nous donnerons des conditions de qualification uniformes assurant la stabilité de la slice convergence d'ensembles vis-à-vis d'une classe d'opérateurs linéaires. Notons que ce problème avait été étudié par Mc Linden et Bergstrom en dimension finie avec l'épi-convergence qui coïncide dans ce cas avec la slice convergence. Le résultat clé dans leur étude est le suivant ([30], Théor. 3, p. 130) : si $A_{n}, n \in \mathbb{N}, A: \mathbb{R}^{p} \longrightarrow \mathbb{R}^{q}$ sont des opérateurs linéaires tels que $A_{n} \longrightarrow A$ et $C_{n}, n \in \mathbb{N}, C$ sont dans $\mathcal{C}\left(\mathbb{R}^{p}\right)$ et vérifient $C_{n} \stackrel{P . K}{\longrightarrow} C$ et $\operatorname{Ker} A \cap C^{\infty}=\{\theta\}$, alors $A_{n}\left(C_{n}\right) \in \mathcal{C}\left(\mathbb{R}^{q}\right)$ pour $n$ assez grand et $A_{n}\left(C_{n}\right) \stackrel{P . K}{\longrightarrow} A(C)$; où $\operatorname{Ker} A$ désigne le noyau de $A$.

Ce résultat tombe en défaut en dimention infinie avec la slice convergence :

Exemple 3.1. Soit $X$ l'espace de Hilbert $L^{2}([0,2 \pi])$. Pour tout $n \geq 1$ posons $A_{n}:=A: f \in X \longmapsto$ $\int_{0}^{2 \pi} x f(x) \mathrm{d} x$ et $C_{n}:=\left\{\alpha e_{n} / \alpha \in \mathbb{R}\right\}$ où $e_{n}(x):=\sin n x$. Il est clair que $C_{n} \stackrel{M}{\longrightarrow} C:=\{\theta\}$ et donc Ker $A \cap C^{\infty}=$ $\{\theta\}$, mais $A_{n}\left(C_{n}\right)$ ne converge pas au sens de Mosco vers $A(C)$, car $A_{n}\left(C_{n}\right)=\mathbb{R}$ et $A(C)=\{\theta\}$.

Théorème 3.2. Soient $X$ et $Y$ deux espaces de Banach. Soient $A_{n}, n \in I N, A: X \longrightarrow Y$ des opérateurs linéaires continus tels que $A_{n} \stackrel{U B}{\longrightarrow} A$, et $C_{n}, n \in I N, C$ des ensembles de $\mathcal{C}(X)$ tels que $C_{n} \stackrel{\tau_{s}}{\longrightarrow} C$. Supposons que l'hypothèse $(H)$ soit vérifiée :

$(H):\left\{\begin{array}{c}\text { Pour toute sous-suite }\left(n_{k}\right)_{k} \text { et toute suite }\left(x_{k}\right)_{k} \text { telles que } x_{k} \in C_{n_{k}} \\ \text { et } \sup _{k}\left\|A_{n_{k}}\left(x_{k}\right)\right\|<+\infty,\left(x_{k}\right)_{k} \text { admet une sous-suite w-convergente. }\end{array}\right.$

Alors $\mathrm{cl}\left(A_{n}\left(C_{n}\right)\right) \stackrel{\tau_{s}}{\longrightarrow} A(C)$ où $\mathrm{cl}\left(A_{n}\left(C_{n}\right)\right)$ désigne la fermeture de l'ensemble $A_{n}\left(C_{n}\right)$. En particulier $(H)$ est satisfaite si l'hypothèse $\left(H^{\prime}\right)$ est vérifiée :

$\left(H^{\prime}\right):\left\{\begin{array}{c}\text { Pour toute sous-suite }\left(n_{k}\right)_{k} \text { et toute suite }\left(x_{k}\right)_{k} \text { telles que } x_{k} \in C_{n_{k}} \\ \text { et } \sup _{k}\left\|A_{n_{k}}\left(x_{k}\right)\right\|<+\infty, \text { il existe un convexe fermé } D \pi-\text { localement } \\ \text { compact contenant la suite }\left(x_{k}\right)_{k} \text { et vérifiant Ker } A \cap D^{\infty}=\{\theta\} .\end{array}\right.$

Pour la preuve de ce théorème, nous aurons besoin du lemme suivant dont le rôle est fondamental dans la suite de cet article. Sa version originale se trouve dans les références $[17,18]$. Nous la modifions légèrement en vue de l'adapter à notre étude.

Lemme 3.3. Soient $X$ un e.l.c.s. et $D$ un convexe fermé localement compact contenant $\theta$. Soit $V$ un voisinage de $\theta$ convexe fermé équilibré tel que $V \cap D$ soit un compact et $j$ la jauge de $V$. Alors pour toute suite $\left(x_{n}\right)_{n}$ de $D$ nous avons :

- ou bien $\left(j\left(x_{n}\right)\right)_{n}$ est bornée et $\left(x_{n}\right)_{n}$ est relativement compacte;

- ou bien $\left(j\left(x_{n}\right)\right)_{n}$ n'est pas bornée et $\left(\frac{x_{n}}{j\left(x_{n}\right)}\right)_{n}$ admet alors une valeur d'adhérence non nulle $y \in D^{\infty}$.

Démonstration. La preuve de ce lemme est quasi-identique à celle du lemme I.8 de [17]. Nous la reprenons pour l'intérêt considérable qu'elle présente dans les démonstrations des résultats de cet article.

Soit $\left(x_{n}\right)_{n} \subset D$. Si $\left(j\left(x_{n}\right)\right)_{n}$ est bornée, il existe $\alpha>1$ tel que $j\left(x_{n}\right)<\alpha, \forall n$. Par suite $j\left(\frac{x_{n}}{\alpha}\right)<1$ et $x_{n} \in(\alpha V) \cap D=\alpha\left(V \cap \frac{D}{\alpha}\right) \subset \alpha(V \cap D)$ car $D$ est un convexe contenant $\theta$. Ainsi, $x_{n} \in \alpha(V \cap D)$ qui est compact; donc $\left(x_{n}\right)_{n}$ est relativement compacte. Si $\left(j\left(x_{n}\right)\right)_{n}$ n'est pas bornée, on peut supposer que $j\left(x_{n}\right) \longrightarrow+\infty$ pour une sous-suite et donc $j\left(x_{n}\right)>1$ pour $n$ suffisamment grand. Ainsi,

$$
\frac{x_{n}}{j\left(x_{n}\right)} \in\left(V \backslash \frac{1}{2} V\right) \cap D \subset\left(V \backslash \frac{1}{2} \operatorname{int}(V)\right) \cap D=: B \subset V \cap D,
$$


où $\operatorname{int}(V)$ désigne l'intérieur de $V$. Donc $B$ est un compact ne contenant pas $\theta$. Ainsi $\frac{x_{n}}{j\left(x_{n}\right)}$ admet une valeur d'adhérence non nulle $y \in B$. Soit maintenant $\varepsilon>0$. Alors pour $n$ suffisamment grand $j\left(x_{n}\right)>\varepsilon$ et donc $\varepsilon \frac{x_{n}}{j\left(x_{n}\right)} \in D$, i.e. $\frac{x_{n}}{j\left(x_{n}\right)} \in \frac{D}{\varepsilon}$, d'où $y \in \frac{D}{\varepsilon}, \forall \varepsilon>0$. Donc $y \in \cap_{\varepsilon>0} \frac{D}{\varepsilon}=D^{\infty}$.

Remarque 3.4. Tout au long de cet article nous allons utiliser le résultat du lemme précédent de manière explicite ou implicite. Si $X$ est un espace de Banach et si $D$ est un convexe fermé contenant $\theta$ et $\pi$-localement compact, alors en se référant à la démonstration du lemme précédent, nous remarquons que si $\left(x_{n}\right)_{n}$ est une suite de $D$ telle que $\left(j\left(x_{n}\right)\right)_{n}$ soit bornée alors $\left(x_{n}\right)_{n}$ est $\pi$-relativement compacte. Elle est donc $w$-relativement compacte. Comme les $w$-compacts sont aussi séquentiellement faiblement compacts [27], alors $\left(x_{n}\right)_{n}$ admet une sous-suite qui converge faiblement vers un élément de $D$. Si $\left(j\left(x_{n}\right)\right)_{n}$ n'est pas bornée, on conclut comme précédement que $\left(\frac{x_{n}}{j\left(x_{n}\right)}\right)_{n}$ admet une sous-suite qui converge faiblement vers un élément de $D^{\infty} \backslash\{\theta\}$.

Démonstration du théorème 3.2. Nous allons procéder en deux étapes :

Étape 1. Prouvons tout d'abord que $A_{n}\left(C_{n}\right) \underset{M}{M} A(C)$ :

(i) soit $x \in C$. Comme $C_{n} \stackrel{\tau_{s}}{\longrightarrow} C$ alors $C_{n} \stackrel{\vec{M}}{\longrightarrow} C$ et donc il existe $x_{n} \in C_{n}$ tel que $x_{n} \stackrel{\|\cdot\|}{\longrightarrow} x$. Ainsi, $A_{n}\left(x_{n}\right) \in A_{n}\left(C_{n}\right)$ et $A_{n}\left(x_{n}\right) \stackrel{\|\cdot\|}{\longrightarrow} A(x)$ car $A_{n} \stackrel{U B}{\longrightarrow} A$ et $A$ est continu;

(ii) si $A_{n_{k}}\left(t_{k}\right) \stackrel{w}{\longrightarrow} z$ pour une sous-suite $\left(n_{k}\right)_{k}$ telle que $t_{k} \in C_{n_{k}}$, alors la suite $\left(A_{n_{k}}\left(t_{k}\right)\right)_{k}$ est bornée et $t_{k} \stackrel{w}{\longrightarrow} t$ pour une sous-suite d'après $(\mathrm{H})$. Il en résulte que $t \in C$; et pour tout $y^{*} \in Y^{*}$ nous avons :

$$
\begin{aligned}
\left|\left\langle A_{n_{k}}\left(t_{k}\right), y^{*}\right\rangle-\left\langle A(t), y^{*}\right\rangle\right| & \leq\left|\left\langle A_{n_{k}}\left(t_{k}\right), y^{*}\right\rangle-\left\langle A\left(t_{k}\right), y^{*}\right\rangle\right|+\left|\left\langle A\left(t_{k}\right), y^{*}\right\rangle-\left\langle A(t), y^{*}\right\rangle\right| \\
& \leq\left\|A_{n_{k}}\left(t_{k}\right)-A\left(t_{k}\right)\right\|\left\|y^{*}\right\|_{*}+\left|\left\langle A\left(t_{k}\right), y^{*}\right\rangle-\left\langle A(t), y^{*}\right\rangle\right| .
\end{aligned}
$$

Cette dernière quantité tend vers 0 quand $k \longrightarrow+\infty$ car $A_{n_{k}} \stackrel{U B}{\longrightarrow} A$ et $A$ est $(w, w)$-continu [16]. Ainsi, $A_{n_{k}}\left(t_{k}\right) \stackrel{w}{\longrightarrow} z=A(t) \in A(C)$. Donc $A_{n}\left(C_{n}\right) \stackrel{M}{\longrightarrow} A(C)$, et par suite $A(C)$ est fermé [1].

Étape 2. Montrons que $c l\left(A_{n}\left(C_{n}\right)\right) \stackrel{\tau_{s}}{\longrightarrow} A(C)$.

(i) Si $O$ est un $\|$. $\|$-ouvert de $Y$ tel que $A(C) \cap O \neq \emptyset$, alors d'après l'étape 1-(i) $A_{n}\left(C_{n}\right) \cap O \neq \emptyset$ et donc $c l\left(A_{n}\left(C_{n}\right)\right) \cap O \neq \emptyset$ pour $n$ assez grand.

(ii) Supposons maintenant que $A(C) \in\left(B^{c}\right)^{++}$où $B$ est un convexe \|.\|-fermé et borné de $Y$. Par l'absurde, s'il existe une sous-suite $\left(n_{k}\right)_{k}$ telle que $\operatorname{cl}\left(A_{n_{k}}\left(C_{n_{k}}\right)\right) \notin\left(B^{c}\right)^{++}$, alors pour chaque $k$ nous avons

$$
c l\left(A_{n_{k}}\left(C_{n_{k}}\right)\right)+\varepsilon_{k} U \not \subset B^{c} \text { avec } \varepsilon_{k} \longrightarrow 0 \text { quand } k \longrightarrow+\infty .
$$

Il existe alors une suite $v_{k} \in\left(\operatorname{cl}\left(A_{n_{k}}\left(C_{n_{k}}\right)\right)+\varepsilon_{k} U\right) \cap B$, et partant une suite $z_{k}:=A_{n_{k}}\left(t_{k}\right), t_{k} \in C_{n_{k}}$ telles que $\left\|v_{k}-z_{k}\right\| \longrightarrow 0$. Comme $\left(v_{k}\right)_{k}$ est bornée, $\left(z_{k}\right)_{k}$ l'est aussi et $t_{k} \stackrel{w}{\longrightarrow} t \in C$ pour une sous-suite d'après (H); et comme dans l'étape 1-(ii) $z_{k} \stackrel{w}{\longrightarrow} A(t)$. Ainsi, $v_{k} \stackrel{w}{\longrightarrow} A(t) \in A(C) \cap B$ ce qui est absurde car $A(C) \subset B^{c}$. Donc $c l\left(A_{n}\left(C_{n}\right)\right) \in\left(B^{c}\right)^{++}$pour $n$ suffisamment grand et par suite $c l\left(A_{n}\left(C_{n}\right)\right) \stackrel{\tau_{s}}{\longrightarrow} A(C)$ d'après (i).

En particulier si l'hypothèse (H') est vérifiée alors (H) l'est aussi. En effet, soit $\left(n_{k}\right)_{k}$ une sous-suite telle que $x_{k} \in C_{n_{k}}$ et $\sup _{k}\left\|A_{n_{k}}\left(x_{k}\right)\right\|<+\infty$. Notons $j$ la jauge d'un $\pi$-voisinage $V$ de $\theta$ convexe fermé équilibré tel que $V \cap\left(D-x_{0}\right)$ soit $\pi$-compact, avec $x_{0}$ un point quelconque de $D$. Si $\left(j\left(x_{k}-x_{0}\right)\right)_{k}$ n'est pas bornée (quitte à supposer que $j\left(x_{k}-x_{0}\right) \longrightarrow+\infty$ quand $\left.k \longrightarrow+\infty\right)$ alors le lemme 3.3 et la remarque 3.4 impliquent l'existence d'une sous-suite $\left(x_{k_{p}}-x_{0}\right)_{p}$ telle que la suite $z_{p}:=\frac{x_{k_{p}}-x_{0}}{j\left(x_{k_{p}}-x_{0}\right)}$ soit $w$-convergente vers un élément $z \in D^{\infty} \backslash\{\theta\}$. Comme $\left(A_{n_{k_{p}}}\left(x_{k_{p}}-x_{0}\right)\right)_{p}$ est bornée alors $A(z)=\theta$. Par suite $z \in \operatorname{Ker} A \cap D^{\infty}=\{\theta\}$, ce qui est absurde. Par conséquent, $\left(j\left(x_{k}-x_{0}\right)\right)_{k}$ est bornée et $\left(x_{k}\right)_{k}$ admet une sous-suite $w$-convergente d'après la remarque 3.4. Ce qui achève la preuve.

Corollaire 3.5. Sous les hypothèses du théorème 3.2 sauf que (H) est remplacée par

$\left(H^{\prime \prime}\right):\left\{\begin{array}{c}\text { Il existe un convexe fermé } D \pi \text { - localement compact et } \\ N \in I N \text { tels que } C_{n} \subset D, \forall n \geq N \text { et } \operatorname{Ker} A \cap D^{\infty}=\{\theta\} .\end{array}\right.$

$A_{n}\left(C_{n}\right)$ est fermé pour $n$ suffisamment grand et $A_{n}\left(C_{n}\right) \stackrel{\tau_{s}}{\longrightarrow} A(C)$. 
Pour la preuve de ce corollaire, nous utiliserons le lemme suivant :

Lemme 3.6 [34]. Soient $X$ et $Y$ deux e.l.c.s., $A: X \longrightarrow Y$ un opérateur linéaire continu et $C$ un convexe fermé de $X$, localement compact. Supposons que $\operatorname{Ker} A \cap C^{\infty}$ soit un sous-espace vectoriel. Alors $A(C)$ est fermé.

Démonstration du corollaire 3.5. D'après le théorème 3.2, nous avons

$$
c l\left(A_{n}\left(C_{n}\right)\right) \stackrel{\tau_{s}}{\longrightarrow} A(C)=\operatorname{cl}(A(C))
$$

puisque l'hypothèse (H') est satisfaite. Il suffit, d'après le lemme précédent, de montrer que $\operatorname{Ker} A_{n} \cap C_{n}^{\infty}=\{\theta\}$ pour $n$ suffisamment grand. Par l'absurde, supposons qu'il existe une sous-suite $\left(n_{k}\right)_{k}$ et une suite $\left(z_{k}\right)_{k}$ telles que pour tout $k \geq 1$ on ait $z_{k} \neq \theta$ et $z_{k} \in \operatorname{Ker} A_{n_{k}} \cap C_{n_{k}}^{\infty}$. Le fait que $z_{k}$ soit non nul implique que $j\left(z_{k}\right)>0$ ( $j$ étant la jauge du voisinage $V$ de $\theta$ considéré dans la preuve du théorème précédent). En effet, s'il existe un indice $k_{0}$ tel que $j\left(z_{k_{0}}\right)=0$ alors $z_{k_{0}} \in V^{\infty}$. Comme $C_{n_{k_{0}}}$ est un convexe fermé $\subset D$ alors $C_{n_{k_{0}}}^{\infty} \subset D^{\infty}$. Ainsi, $z_{k_{0}} \in V^{\infty} \cap D^{\infty}=\left(V \cap\left(D-x_{0}\right)\right)^{\infty}=\{\theta\}$, car $V \cap\left(D-x_{0}\right)$ est $\pi$-compact. Ceci est impossible car $z_{k_{0}} \neq \theta$. En outre nous avons $\frac{z_{k}}{j\left(z_{k}\right)} \in\left(V \backslash \frac{1}{2} \operatorname{int}(V)\right) \cap D^{\infty} \subset\left(V \backslash \frac{1}{2} \operatorname{int}(V)\right) \cap\left(D-x_{0}\right)$ qui est faiblement compact; donc $\frac{z_{k}}{j\left(z_{k}\right)} \stackrel{w}{\longrightarrow} z \in D^{\infty}$ pour une sous-suite avec $z \neq \theta$. D'autre part, comme $\frac{z_{k}}{j\left(z_{k}\right)} \in \operatorname{Ker} A_{n_{k}}$ et que $A_{n} \stackrel{U B}{\longrightarrow} A$ alors $A(z)=\theta$, et par suite $z \in \operatorname{Ker} A \cap D^{\infty}=\{\theta\}$. Ce qui est absurde.

Corollaire 3.7. Sous les hypothèses du corollaire 3.5 avec $D:=\cup_{\lambda \geq 0} \lambda K$ où $K$ est un convexe $\pi$-compact vérifiant $\operatorname{Ker} A \cap K=\emptyset$, les ensembles $A_{n}\left(C_{n}\right)$ sont fermés pour $n$ suffisamment grand et $A_{n}\left(C_{n}\right) \stackrel{\tau_{s}}{\longrightarrow} A(C)$.

Démonstration. L'ensemble $D=\cup_{\lambda \geq 0} \lambda K$ est un cône convexe. Comme $K$ est convexe $\pi$-compact et $\theta \notin K, D$ est alors fermé et $\pi$-localement compact [27], p. 338. De plus l'hypothèse $\operatorname{Ker} A \cap K=\emptyset$ implique clairement que $\operatorname{Ker} A \cap D^{\infty}=\operatorname{Ker} A \cap D=\{\theta\}$. Le corollaire 3.5 achève la preuve.

Corollaire 3.8 [30]. Soient $A_{n}, n \in I N, A: \mathbb{R}^{p} \longrightarrow \mathbb{R}^{q}$ des opérateurs linéaires tels que $A_{n} \longrightarrow A$ et $C_{n}$, $n \in I N, C$ des ensembles de $\mathcal{C}\left(\mathbb{R}^{p}\right)$ tels que $C_{n} \stackrel{P . K}{\longrightarrow} C$ et $\operatorname{Ker} A \cap C^{\infty}=\{\theta\}$. Alors $A_{n}\left(C_{n}\right), A(C)$ sont dans $\mathcal{C}\left(\mathbb{R}^{q}\right)$ pour tout $n$ suffisamment grand et $A_{n}\left(C_{n}\right) \stackrel{P . K}{\longrightarrow} A(C)$.

Démonstration. En dimension finie la slice convergence d'ensembles est identique à la convergence au sens de Painlevé-Kuratowski [10]. Dans ce cas aussi, il est classique de vérifier que la convergence simple de la suite $\left(A_{n}\right)_{n}$ vers $A$ est équivalente à sa convergence uniforme sur les bornés de $\mathbb{R}^{p}$. Il suffit alors de prouver que la condition $\operatorname{Ker} A \cap C^{\infty}=\{\theta\}$ implique l'hypothèse $(\mathrm{H})$ du théorème 3.2. En effet : soit $\left(n_{k}\right)_{k}$ une sous-suite et $\left(x_{k}\right)_{k}$ une suite vérifiant $x_{k} \in C_{n_{k}}$ pour tout $k$ et $\sup _{k}\left\|A_{n_{k}}\left(x_{k}\right)\right\|<+\infty$. Puisque $C_{n} \stackrel{P . K}{\longrightarrow} C$, on peut supposer sans nuire à la généralité que $\theta \in C_{n} \cap C$. Si $\left(x_{k}\right)_{k}$ n'est pas bornée, quitte à extraire une sous-suite, on peut supposer que $\left\|x_{k}\right\| \longrightarrow+\infty$. Soient $\varepsilon>0$ et $k$ suffisamment grand tels que $\left\|x_{k}\right\|>\varepsilon$. D'après la convexité des $C_{n}, \frac{\varepsilon x_{k}}{\left\|x_{k}\right\|} \in C_{n_{k}}$ et donc il existe une valeur d'adherence $\bar{x}$ de la suite $\frac{x_{k}}{\left\|x_{k}\right\|}$ telle que $\varepsilon \bar{x} \in C$ et $A(\bar{x})=\theta$, i.e. $\bar{x} \in \operatorname{Ker} A \cap C^{\infty}=\{\theta\}$; ce qui est absurde car $\|\bar{x}\|=1$. Donc $\left(x_{k}\right)_{k}$ est bornée, ce qui achève la preuve.

Remarque 3.9. En dimension finie la condition $\operatorname{Ker} A \cap C^{\infty}=\{\theta\}$ n'implique pas en général l'existence d'un convexe fermé $D$ contenant les ensembles $C_{n}$ et $\operatorname{Ker} A \cap D^{\infty}=\{\theta\}$. En effet, soient $X:=\mathbb{R}^{2}$ et $Y:=\mathbb{R}$. Définissons les ensembles $C_{n}:=\{(x, y) / y=n x\}, C:=\{0\} \times \mathbb{R}$ et les opérateurs $A_{n}(x, y):=A(x, y):=y$. Il est clair que conv $\left(C_{n} \cup C_{p}\right)=\mathbb{R}^{2}$ si $n \neq p$, et donc s'il existe un convexe fermé $D$ tel que $C_{n} \subset D$ pour $n$ suffisamment grand alors $D=\mathbb{R}^{2}$. D'autre part, nous avons $C_{n} \stackrel{P . K}{\longrightarrow} C$ et $\operatorname{Ker} A \cap C^{\infty}=\{\theta\}=\{(0,0)\}$ mais $\operatorname{Ker} A \cap D^{\infty}=\operatorname{Ker} A \neq\{\theta\}$.

Nous donnons maintenant des conditions de qualification uniformes assurant la stabilité de la slice convergence vis-à-vis de l'addition d'ensembles de $\mathcal{C}(X)$. Notons que ce problème avait été étudié aussi par Mc Linden et Bergstrom [30] en dimension finie. Leur résultat est le suivant : Si $C, C_{n}$ et $D, D_{n}$ sont des ensembles de $\mathcal{C}\left(\mathbb{R}^{p}\right)$ vérifiant $C_{n} \stackrel{P . K}{\longrightarrow} C, D_{n} \stackrel{P . K}{\longrightarrow} D$ et $C^{\infty} \cap-D^{\infty}=\{\theta\}$, alors $C_{n}+D_{n} \in \mathcal{C}\left(\mathbb{R}^{p}\right)$ pour tout $n$ suffisamment grand 
et $C_{n}+D_{n} \stackrel{P . K}{\longrightarrow} C+D$. Ce résultat n'est plus vrai en dimension infinie lorsqu'on considère la convergence au sens de Painlevé-Kuratowski ou la slice convergence.

Exemple 3.10. Soit $X$ un espace de Hilbert de base orthonormale $\left(e_{n}\right)_{n \geq 1}$.

a) Considérons les segments : $C_{n}:=\left[e_{1}, e_{n}\right], D_{n}:=\left[e_{1},-e_{n}\right]$ et $C:=D:=\left\{e_{1}\right\}$. Il est clair que $C_{n} \stackrel{P . K}{\longrightarrow} C$, $D_{n} \stackrel{P . K}{\longrightarrow} D$ et $C^{\infty} \cap-D^{\infty}=\{\theta\}$. Mais $C_{n}+D_{n} \stackrel{P . K}{\longrightarrow} C+D$ car $\left[\theta, 2 e_{1}\right] \subset C_{n}+D_{n}$ pour tout $n$.

b) Considérons les ensembles : $A_{n}:=\left[e_{n}, v_{n}\right]=\left\{\alpha e_{n}+(1-\alpha) v_{n} / 0 \leq \alpha \leq 1\right\}$ avec $v_{n}:=\frac{e_{n}+e_{n+1}}{n}, B_{n}:=$ $\operatorname{esp}\left\{e_{1}, \ldots, e_{n}\right\}$ le s.e.v. engendré par $\left(e_{k}\right)_{1 \leq k \leq n}$. Il est clair que $A_{n} \stackrel{M}{\longrightarrow} A:=\{\theta\}$ et $B_{n} \stackrel{M}{\longrightarrow} B:=X$. Maintenant soient les fonctions : $f_{n}(x):=I_{A_{n}}^{*}(x)=\sup _{t \in A_{n}}\langle t, x\rangle=\operatorname{Max}\left\{\left\langle e_{n}, x\right\rangle,\left\langle v_{n}, x\right\rangle\right\}, g_{n}(x):=I_{B_{n}}^{*}(x)=I_{B_{n}^{\perp}}(x)$ où $B_{n}^{\perp}:=\operatorname{esp}\left\{e_{n+1}, \ldots\right\}$ le s.e.v. fermé engendré par $\left(e_{k}\right)_{k \geq n+1}$. Nous savons que $f_{n} \stackrel{M}{\longrightarrow} f:=I_{A}^{*} \equiv 0$ et $g_{n} \stackrel{M}{\longrightarrow} g:=$ $I_{B}^{*}=I_{\{\theta\}}$. Considérons alors les ensembles de $X \times \mathbb{R}: C_{n}:=$ epi $f_{n}, D_{n}:=$ epi $g_{n}, C:=$ epif=X $f \times \mathbb{R}^{+}$et $D:=e p i g=\{\theta\} \times \mathbb{R}^{+}$. Nous avons $C_{n} \stackrel{M}{\longrightarrow} C$ et $D_{n} \stackrel{M}{\longrightarrow} D$. D'autre part, les deux suites $z_{n}:=-n e_{n+1}-e_{n}$ et $y_{n}:=n e_{n+1}$ vérifient $f_{n}\left(z_{n}\right)=-1$ et $g_{n}\left(y_{n}\right)=0$. Il en résulte que $\left(z_{n},-1\right) \in C_{n}$ et $\left(y_{n}, 0\right) \in D_{n}$. Ainsi, $\left(z_{n}+y_{n},-1\right)=\left(-e_{n},-1\right) \in C_{n}+D_{n}$ et $\left(-e_{n},-1\right) \stackrel{w \times|.|}{\longrightarrow}(\theta,-1) \notin C+D=X \times \mathbb{R}^{+}$. D'où, $C_{n}+D_{n} \stackrel{M}{\longleftrightarrow} C+D$ bien que $C^{\infty} \cap-D^{\infty}=C \cap-D=\{(\theta, 0)\}$.

Les trois théorèmes qui suivent constituent un résultat central dans la suite de cet article et sont à la base de la version fonctionnelle de la stabilité.

Théorème 3.11. Soient $X$ un espace de Banach et $C_{n}, D_{n}, n \geq 1, C, D$ des ensembles de $\mathcal{C}(X)$ tels que $C_{n} \stackrel{\tau_{s}}{\longrightarrow} C$ et $D_{n} \stackrel{\tau_{s}}{\longrightarrow} D$. Supposons qu'il existe $C_{0}$ et $D_{0}$ deux ensembles de $\mathcal{C}(X)$ vérifiant $C_{0}^{\infty} \cap-D_{0}^{\infty}=\{\theta\}$, $D_{0}$ est $\pi$-localement compact et $C_{n} \subset C_{0}, D_{n} \subset D_{0}$ pour tout $n \geq \grave{a}$ un certain $N$. Alors $C_{n}+D_{n}, C+D$ sont dans $\mathcal{C}(X)$ pour tout $n \geq N$ et $C_{n}+D_{n} \stackrel{\tau_{s}}{\longrightarrow} C+D$.

En considérant l'exemple 3.10-b), nous remarquons que la conclusion du théorème 3.11 n'est plus vraie lorsque nous avons seulement $C_{n} \stackrel{\tau_{s}}{\longrightarrow} C, D_{n} \stackrel{\tau_{s}}{\longrightarrow} D$ avec $C^{\infty} \cap-D^{\infty}=\{\theta\}$ et $D$ est $\pi$-localement compact !

Pour la preuve de ce théorème on ne peut pas appliquer le corollaire 3.5 avec $A_{n}(x, y):=A(x, y):=x+y$, $(x, y) \in X^{2}$ et $C_{n} \times D_{n} \subset C_{0} \times D_{0}$ puisque $C_{0} \times D_{0}$ n'est pas en général $\pi$-localement compact. Pour cela nous allons utiliser le lemme suivant :

Lemme 3.12. Soient $X$ un espace de Banach, $C_{n}, n \in I N, C$ des ensembles de $\mathcal{C}(X)$ et $f_{n}, n \in I N, f$ des fonctions de $\Gamma(X)$. Alors

(i) $C_{n} \stackrel{\tau_{s}}{\longrightarrow} C \Longrightarrow \forall x \in X, \forall x_{n} \stackrel{\|\cdot\|}{\longrightarrow} x, C_{n}-x_{n} \stackrel{\tau_{s}}{\longrightarrow} C-x$.

(ii) Si $\exists x \in X, \exists x_{n} \stackrel{\|\cdot\|}{\longrightarrow} x, C_{n}-x_{n} \stackrel{\tau_{s}}{\longrightarrow} C-x$ alors $C_{n} \stackrel{\tau_{s}}{\longrightarrow} C$.

(iii) $f_{n} \stackrel{\tau_{s}}{\longrightarrow} f \Longleftrightarrow \exists x \in X, \exists x_{n} \stackrel{\|\cdot\|}{\longrightarrow} x, f_{n}\left(.+x_{n}\right) \stackrel{\tau_{s}}{\longrightarrow} f(.+x)$.

Démonstration. (i) Supposons que $C_{n} \stackrel{\tau_{s}}{\longrightarrow} C$. Comme $C_{n} \stackrel{M}{\longrightarrow} C$, alors $\forall x \in X, \forall x_{n} \stackrel{\|\cdot\|}{\longrightarrow} x, C_{n}-x_{n} \stackrel{M}{\longrightarrow} C-x$. Ainsi $\forall u \in X, \exists u_{n} \stackrel{\|\cdot\|}{\longrightarrow} u$ telle que $\overrightarrow{I_{C_{n}-x_{n}}}\left(u_{n}\right) \longrightarrow I_{C-x}(u)$. Soient maintenant $(y, \eta) \in X^{*} \times \mathbb{R}$ tel que $\eta>I_{C-x}^{*}(y)$, i.e. $\eta>\widehat{I_{C}^{*}}(y)-\langle x, y\rangle, \delta \in \mathbb{R}$ tel que $\eta+\langle x, y\rangle>\delta>I_{C}^{*}(y)$ et $\left(v_{n}\right)_{n}$ une suite bornée de $X$. Puisque $I_{C_{n}} \stackrel{\tau_{s}}{\longrightarrow} I_{C}$ alors nous avons

$$
\begin{aligned}
I_{C_{n}}\left(v_{n}+x_{n}\right) & >\left\langle v_{n}+x_{n}, y\right\rangle-\delta \\
& >\left\langle v_{n}, y\right\rangle+\left\langle x_{n}, y\right\rangle-\eta-\left\langle x_{n}, y\right\rangle
\end{aligned}
$$

pour tout $n$ suffisamment grand, i.e. $I_{C_{n}}\left(v_{n}+x_{n}\right)>\left\langle v_{n}, y\right\rangle-\eta$ ou encore

$$
I_{C_{n}-x_{n}}\left(v_{n}\right)>\left\langle v_{n}, y\right\rangle-\eta
$$

Par conséquent, $I_{C_{n}-x_{n}} \stackrel{\tau_{s}}{\longrightarrow} I_{C-x}$ et donc $C_{n}-x_{n} \stackrel{\tau_{s}}{\longrightarrow} C-x$ [10].

(ii) Si $C_{n}-x_{n} \stackrel{\tau_{s}}{\longrightarrow} C-x$ avec $x_{n} \stackrel{\|\cdot\|}{\longrightarrow} x$ alors d'après (i)

$$
C_{n}-x_{n}-\left(-x_{n}\right) \stackrel{\tau_{s}}{\longrightarrow} C-x-(-x), \text { i.e. } C_{n} \stackrel{\tau_{s}}{\longrightarrow} C \text {. }
$$


(iii) Elle est immédiate à partir de (i) et (ii) puisque, epi $f_{n}\left(.+x_{n}\right)=$ epi $f_{n}-\left(x_{n}, 0\right)$ et epi $f(.+x)=$ epi $f-(x, 0)$.

Remarque 3.13. Le résultat du lemme précédent reste valable lorsqu'on considère la slice convergence duale d'ensembles et de fonctions. La preuve est identique à la précédente moyennant [10], Corollaire 3.6 ii).

Démonstation du théorème 3.11. D'après le lemme 3.12, vu que $C_{n} \stackrel{\tau_{s}}{\longrightarrow} C$ et $D_{n} \stackrel{\tau_{s}}{\longrightarrow} D$, on peut supposer sans nuire à la généralité que $\theta \in C \cap D$ et $\theta \in C_{n} \cap D_{n}$. Maintenant, puisque $C_{n} \subset \overrightarrow{C_{0}}, D_{n} \subset D_{0}$ alors $D_{n}$ et $D$ sont $\pi$-localement compacts et $C_{n}^{\infty} \cap-D_{n}^{\infty}=C^{\infty} \cap-D^{\infty}=\{\theta\}$. Ainsi, $C_{n}+D_{n}$ et $C+D$ sont dans $\mathcal{C}(X)$ d'après [17].

Montrons maintenant que $C_{n}+D_{n} \stackrel{\tau_{s}}{\longrightarrow} C+D$ :

(i) soit $(x, y) \in C \times D$ et $z:=x+y$. Il existe $x_{n} \in C_{n}$ et $y_{n} \in D_{n}$ tels que $x_{n} \stackrel{\|\cdot\|}{\longrightarrow} x$ et $y_{n} \stackrel{\|\cdot\|}{\longrightarrow} y$. Ainsi $z_{n}:=x_{n}+y_{n} \in C_{n}+D_{n}$ et $z_{n} \stackrel{\|\cdot\|}{\longrightarrow} z$. Donc si $O$ est un $\|$.$\| -ouvert de X$ tel que $(C+D) \cap O \neq \emptyset$ alors $\left(C_{n}+D_{n}\right) \cap O \neq \emptyset$ pour tout $n$ suffisamment grand;

(ii) soit $B$ un convexe fermé borné non vide de $X$ tel que $C+D \in\left(B^{c}\right)^{++}$et soit $\varepsilon_{0}>0$ tel que $C+D+\varepsilon_{0} U \subset$ $B^{c}$. Par l'absurde, si $C_{n}+D_{n} \notin\left(B^{c}\right)^{++}$pour une sous-suite d'ensembles notée encore $C_{n}+D_{n}$ alors il existe une suite $\left(\varepsilon_{n}\right)_{n} \downarrow 0$ telle que $\left(C_{n}+D_{n}+\varepsilon_{n} U\right) \cap B \neq \emptyset$, i.e. il existe $\left(x_{n}, y_{n}\right) \in C_{n} \times D_{n}$ et $v_{n} \stackrel{\|\cdot\|}{\longrightarrow} \theta$ vérifiant $x_{n}+y_{n}+v_{n} \in B$ pour tout $n$.

Soit $j$ la jauge d'un voisinage $V$ de $\theta$ convexe fermé et équilibré tel que $V \cap D_{0}$ soit $\pi$-compact. Si $\left(j\left(y_{n}\right)\right)_{n}$ n'est pas bornée, $\frac{y_{n}}{j\left(y_{n}\right)} \stackrel{w}{\longrightarrow} y \in D_{0}^{\infty}$ pour une sous-suite avec $y \neq \theta$ d'après le lemme 3.3 et la remarque 3.4 . Comme $B$ est borné, $\frac{x_{n}+y_{n}+v_{n}}{j\left(y_{n}\right)} \stackrel{\|\cdot\|}{\longrightarrow} \theta$, et $\frac{x_{n}}{j\left(y_{n}\right)} \stackrel{w}{\longrightarrow}-y$. Maintenant pour tout $\varepsilon>0$ et tout $n$ suffisamment grand tels que $j\left(y_{n}\right)>\varepsilon$, nous avons $\frac{\varepsilon x_{n}}{j\left(y_{n}\right)} \in C_{n}$ et donc $\frac{\varepsilon x_{n}}{j\left(y_{n}\right)} \stackrel{w}{\longrightarrow}-\varepsilon y \in C$. Ainsi $y \in-C_{0}^{\infty} \cap D_{0}^{\infty}=\{\theta\}$, ce qui est absurde. Donc $\left(j\left(y_{n}\right)\right)_{n}$ est bornée, par suite $\left(y_{n}\right)_{n}$ est bornée et $y_{n} \stackrel{w}{\longrightarrow} y \in D$ pour une sous-suite. On en déduit que $\left(x_{n}\right)_{n}$ est aussi bornée. D'autre part, comme $\left(C+D+\operatorname{int}\left(\varepsilon_{0} U\right)\right) \cap B=\emptyset$ et $C+D+i n t\left(\varepsilon_{0} U\right)$ est un $\|$.\|-ouvert convexe, on peut séparer $B$ et $C+D+\operatorname{int}\left(\varepsilon_{0} U\right)$ par un hyperplan fermé, i.e. il existe $z \in X^{*}$ non nul et $\eta \in \mathbb{R}$ tels que

$$
\sup _{t \in C}\langle t, z\rangle+\sup _{t \in D}\langle t, z\rangle+\varepsilon_{0}\|z\|_{*} \leq \eta \leq \inf _{t \in B}\langle t, z\rangle .
$$

En posant $\delta:=\sup _{t \in C}\langle t, z\rangle$ nous avons d'après les inégalités précédentes

$$
\delta\left\langle\eta-\langle y, z\rangle-\varepsilon_{1} \leq\left\langle x_{n}+y_{n}+v_{n}, z\right\rangle-\langle y, z\rangle-\varepsilon_{1}\right.
$$

où $0<\varepsilon_{1}<\varepsilon_{0}\|z\|_{*}$. D'où

$$
\delta<\eta-\langle y, z\rangle-\varepsilon_{1}:=r<\left\langle x_{n}, z\right\rangle
$$

pour tout $n$ assez grand car $\left\langle y_{n}+v_{n}-y, z\right\rangle \longrightarrow 0$. Ainsi, $I_{C}^{*}(z)<r$, mais $I_{C_{n}}\left(x_{n}\right)=0<\left\langle x_{n}, z\right\rangle-r$. Ceci contredit le fait que $I_{C_{n}} \stackrel{\tau_{s}}{\longrightarrow} I_{C}$. D'où, $C_{n}+D_{n} \in\left(B^{c}\right)^{++}$à partir d'un certain rang. Ce qui achève la preuve du théorème.

Théorème 3.14. Soient $X$ un espace de Banach et $C_{n}, D_{n}, n \geq 1, C, D$ des ensembles de $\mathcal{C}\left(X^{*}\right)$ tels que $C_{n} \stackrel{\tau_{s}^{*}}{\longrightarrow} C$ et $D_{n} \stackrel{\tau_{s}^{*}}{\longrightarrow} D$. Supposons qu'il existe $C_{0}$ et $D_{0}$ deux ensembles de $\mathcal{C}\left(X^{*}\right)$ vérifiant $C_{0}^{\infty} \cap-D_{0}^{\infty}=\left\{\theta^{*}\right\}$, $D_{0}$ est $\pi^{*}$-localement compact et $C_{n} \subset C_{0}, D_{n} \subset D_{0}$ pour tout $n \geq \grave{a ̀ ~ u n ~ c e r t a i n ~} N$. Alors $C_{n}+D_{n}, C+D$ sont dans $\mathcal{C}\left(X^{*}\right)$ et $C_{n}+D_{n} \stackrel{\tau_{s}^{*}}{\longrightarrow} C+D$.

Démonstration. La $w^{*}$-fermeture des $C_{n}+D_{n}$ et $C+D$ se démontre de manière analogue comme dans la preuve du théorème 3.11. D'après la remarque 3.13, on peut supposer sans nuire à la généralité que $\theta^{*} \in C_{n} \cap D_{n} \cap C \cap D$.

(i) Soit $O$ un $\|\cdot\|_{*}$-ouvert de $X^{*}$ tel que $(C+D) \cap O \neq \emptyset$. Alors la condition $\left(C_{n}+D_{n}\right) \cap O \neq \emptyset$ pour tout $n$ assez grand se démontre exactement comme dans (i) de la preuve du théorème 3.11.

(ii) Soit $K$ un convexe $w^{*}$-compact de $X^{*}$ tel que $C+D \in\left(K^{c}\right)^{++}$et soit $\varepsilon_{0}>0$ tel que $C+D+\varepsilon_{0} U^{*} \subset K^{c}$. Par l'absurde si $C_{n}+D_{n} \notin\left(K^{c}\right)^{++}$pour une sous-suite, il existe $\left(\varepsilon_{n}\right)_{n} \downarrow 0$ telle que $\left(C_{n}+D_{n}+\varepsilon_{n} U^{*}\right) \cap K \neq \emptyset$. Donc il existe $\left(x_{n}, y_{n}\right) \in C_{n} \times D_{n}$ et $v_{n} \stackrel{\|\cdot\|_{*}}{\longrightarrow} \theta^{*}$ vérifiant $x_{n}+y_{n}+v_{n} \in K$ pour tout $n$. Soit $j$ la jauge d'un 
voisinage $V$ de $\theta^{*}$ convexe $w^{*}$-fermé et équilibré tel que $V \cap D_{0}$ soit $\pi^{*}$-compact. Si $\left(j\left(y_{n}\right)\right)_{n}$ n'est pas bornée (quitte à supposer que $j\left(y_{n}\right) \longrightarrow+\infty$ pour une sous-suite) alors d'après le lemme $3.3,\left(\frac{y_{n}}{j\left(y_{n}\right)}\right)_{n}$ admet une valeur d'adhérence non nulle $\bar{y}$ pour $\pi^{*}$ (et donc pour $w^{*}$ ) avec $\bar{y} \in D_{0}^{\infty}$. Comme $K$ est borné, $\frac{x_{n}+y_{n}+v_{n}}{j\left(y_{n}\right)} \stackrel{\|\cdot\|_{*}}{\longrightarrow} \theta^{*}$ et donc $\frac{x_{n}}{j\left(y_{n}\right)}+\frac{y_{n}}{j\left(y_{n}\right)} \stackrel{\|\cdot\|_{*}}{\longrightarrow} \theta^{*}$. On en déduit que $\left(\frac{x_{n}}{j\left(y_{n}\right)}\right)_{n}$ admet $-\bar{y}$ comme valeur d'adhérence pour la topologie $w^{*}$. Mais pour tout $\varepsilon>0, \frac{\varepsilon x_{n}}{j\left(y_{n}\right)} \in C_{0}$ pour tout $n$ suffisamment grand. Ainsi, $\varepsilon(-\bar{y}) \in C_{0}$, i.e. $-\bar{y} \in \cap_{\varepsilon>0} \varepsilon C_{0}=C_{0}^{\infty}$. D'où $-\bar{y} \in C_{0}^{\infty} \cap-D_{0}^{\infty}=\left\{\theta^{*}\right\}$, ce qui est absurde. La suite $\left(j\left(y_{n}\right)\right)_{n}$ est donc bornée, et par le lemme 3.3 et [16] la suite $\left(y_{n}\right)_{n}$ est bornée. On en déduit que $\left(x_{n}\right)_{n}$ est aussi bornée. Maintenant, puisque $C+D$ est $w^{*}$-fermé et $U^{*}$ est $w^{*}$-compact alors $C+D+\varepsilon_{0} U^{*}$ est $w^{*}$-fermé d'après [17]. Comme $\left(C+D+\varepsilon_{0} U^{*}\right) \cap K=\emptyset$ et $K$ est convexe $w^{*}$-compact, alors d'après le théorème de Hahn-Banach [15] appliqué dans l'espace $\left(X^{*}, w^{*}\right)$, il existe $x$ non nul dans $X$ et $\eta \in \mathbb{R}$ tels que $\sup _{t \in C+D+\varepsilon_{0} U^{*}}\langle x, t\rangle<\eta<\inf _{t \in K}\langle x, t\rangle$. Par suite,

$$
\sup _{t \in C}\langle x, t\rangle+\sup _{t \in D}\langle x, t\rangle+\varepsilon_{0}\|x\|<\eta<\inf _{t \in K}\langle x, t\rangle \cdot
$$

Ainsi, $I_{C}^{*}(x)$ et $I_{D}^{*}(x)$ sont finis. De plus, pour $r:=\varepsilon_{0}\|x\|+I_{D}^{*}(x)$ nous avons $I_{C}^{*}(x)<\eta-r$. Comme $C_{n} \stackrel{\tau_{s}^{*}}{\longrightarrow} C$, alors $\left.0=I_{C_{n}}\left(x_{n}\right)\right\rangle\left\langle x, x_{n}\right\rangle-(\eta-r)$ pour $n$ suffisamment grand. D'où,

$$
I_{C}^{*}(x)+r<\eta<\left\langle x, x_{n}+y_{n}+v_{n}\right\rangle<\eta-r+\left\langle x, y_{n}+v_{n}\right\rangle \cdot
$$

D'autre part, puisque $D_{n} \stackrel{\tau_{s}^{*}}{\longrightarrow} D$ alors d'après [10], corollaire 3.6, pour tout $\alpha>0$, il existe $N_{\alpha}$ tels que $0=I_{D_{n}}\left(y_{n}\right)>\left\langle x, y_{n}\right\rangle-\alpha-I_{D}^{*}(x)$ pour tout $n \geq N_{\alpha}$. Ainsi,

$$
\left\langle x, y_{n}\right\rangle<\alpha+I_{D}^{*}(x), \forall n \geq N_{\alpha}
$$

On en déduit que $r\left\langle\left\langle x, y_{n}\right\rangle+\left\langle x, v_{n}\right\rangle<2 \alpha+I_{D}^{*}(x)\right.$. Ainsi, $r \leq I_{D^{*}}^{*}(x)$, ce qui est absurde car $x \neq \theta$ et $\varepsilon_{0}>0$. D'où, $C_{n}+D_{n} \in\left(K^{c}\right)^{++}$à partir d'un certain rang, et $C_{n}+D_{n} \stackrel{\tau_{s}^{*}}{\longrightarrow} C+D$.

Théorème 3.15. Soient $X$ et $Y$ deux espaces de Banach, $A_{n}, n \in I N, A: Y^{*} \longrightarrow X^{*}$ des opérateurs linéaires $\left(\|\cdot\|_{*},\|\cdot\|_{*}\right)$ et $\left(w^{*}, w^{*}\right)$-continus tels que $A_{n} \stackrel{U B}{\longrightarrow} A$, et $C_{n}, n \in I N, C$ des ensembles de $\mathcal{C}\left(Y^{*}\right)$ tels que $C_{n} \stackrel{\tau_{s}^{*}}{\longrightarrow} C$. Supposons qu'il existe $D$ convexe $w^{*}$-fermé et $\pi^{*}$-localement compact tel que $C_{n} \subset D$ et $\operatorname{Ker} A \cap D^{\infty}=\left\{\theta^{*}\right\}$. Alors $A_{n}\left(C_{n}\right), A(C)$ sont dans $\mathcal{C}\left(X^{*}\right)$ pour $n$ suffisamment grand et $A_{n}\left(C_{n}\right) \stackrel{\tau_{s}^{*}}{\longrightarrow} A(C)$.

Démonstration. i) Les ensembles $A_{n}\left(C_{n}\right)$ sont évidemment convexes. Pour montrer que $A_{n}\left(C_{n}\right)$ est fermé, il suffit d'après le lemme 3.6 de prouver que $\operatorname{Ker} A_{n} \cap C_{n}^{\infty}=\left\{\theta^{*}\right\}$ pour $n$ suffisamment grand. On peut supposer sans nuire à la généralité que $\theta^{*} \in D$. Soit $j$ la jauge d'un voisinage $V$ de $\theta^{*}$ convexe $w^{*}$-fermé équilibré tel que $V \cap D$ soit $\pi^{*}$-compact. Par l'absurde, supposons que $\operatorname{Ker} A_{n} \cap C_{n}^{\infty}$ contient un élément non nul $y_{n}$ pour une sous-suite. Comme dans la preuve du corollaire 3.5, on montre que $j\left(y_{n}\right)>0$ et la suite $z_{n}:=\frac{y_{n}}{j\left(y_{n}\right)} \in\left(V \backslash \frac{1}{2}\right.$ int $\left.(V)\right) \cap D^{\infty}$ qui est $w^{*}$-compact; elle admet donc une valeur d'adhérence $z \in D^{\infty} \backslash\left\{\theta^{*}\right\}$ pour la topologie $w^{*}$. Or $\left(z_{n}\right)_{n}$ est bornée d'après [16], par conséquent $\left\|A_{n}\left(z_{n}\right)-A\left(z_{n}\right)\right\|_{*} \longrightarrow 0$. Comme $z_{n} \in \operatorname{Ker} A_{n}$ nous avons $\left\|A\left(z_{n}\right)\right\|_{*} \longrightarrow 0$, et donc $A\left(z_{n}\right) \stackrel{w^{*}}{\longrightarrow} \theta^{*}$. Or $A$ est $\left(w^{*}, w^{*}\right)$-continu, il s'en suit que $A(z)$ est une valeur d'adhérence de la suite $\left(A\left(z_{n}\right)\right)_{n}$ pour la topologie $w^{*}$. Par suite, $A(z)=\theta^{*}$. Ainsi, $z \in \operatorname{Ker} A \cap D^{\infty}=\left\{\theta^{*}\right\}$, ce qui est absurde. La fermeture de $A(C)$ provient du fait que $\operatorname{Ker} A \cap C^{\infty}=\left\{\theta^{*}\right\}, C$ est $\pi^{*}$-localement compact est $A$ est $\left(\pi^{*}, w^{*}\right)$-continu.

ii) Montrons que $A_{n}\left(C_{n}\right) \stackrel{\tau_{s}^{*}}{\longrightarrow} A(C)$. Soit $z \in C$, il existe $z_{n} \in C_{n}$ tel que $\left\|z_{n}-z\right\|_{*} \longrightarrow 0$. Donc $\left\|A_{n}\left(z_{n}\right)-A(z)\right\|_{*} \longrightarrow 0$. Par suite, pour tout $\|\cdot\|_{*}$-ouvert $O$ tel que $A(C) \cap O \neq \emptyset$, on a $A_{n}\left(C_{n}\right) \cap O \neq \emptyset$ pour $n$ suffisamment grand. Soit maintenant $K$ un convexe $w^{*}$-compact tel que $A(C) \in\left(K^{c}\right)^{++}$. Il existe $\varepsilon_{0}>0$ tel que $\left(A(C)+\varepsilon_{0} U^{*}\right) \cap K=\emptyset$. Supposons que $A_{n}\left(C_{n}\right) \notin\left(K^{c}\right)^{++}$pour une sous-suite. Alors il existe $\left(\varepsilon_{n}\right)_{n} \downarrow 0$ telle que $\left(A_{n}\left(C_{n}\right)+\varepsilon_{n} U^{*}\right) \cap K \neq \emptyset$. Il existe donc $\left(y_{n}\right) \in C_{n}$ et $v_{n} \stackrel{\|\cdot\|_{*}}{\longrightarrow} \theta^{*}$ tels que $A_{n}\left(y_{n}\right)+v_{n} \in K$ 
pour tout $n$. Ainsi, $\left(A_{n}\left(y_{n}\right)\right)_{n}$ est une suite bornée. Soit $j$ la jauge du voisinage $V$ de $\theta^{*}$ considéré dans i). Si $\left(j\left(y_{n}\right)\right)_{n}$ n'est pas bornée alors $z_{n}:=\frac{y_{n}}{j\left(y_{n}\right)}$ admet une valeur d'adhérence $z \in D^{\infty} \backslash\left\{\theta^{*}\right\}$ pour $w^{*}$. De plus, $\left\|A_{n}\left(\frac{y_{n}}{j\left(y_{n}\right)}\right)-A\left(\frac{y_{n}}{j\left(y_{n}\right)}\right)\right\|_{*} \longrightarrow 0$. Ainsi, $\left\|A\left(z_{n}\right)\right\|_{*} \longrightarrow 0 \operatorname{car}\left(A_{n}\left(y_{n}\right)\right)_{n}$ est bornée et $j\left(y_{n}\right) \longrightarrow+\infty$. On en déduit que $A(z)=\theta^{*}$, i.e. $z \in \operatorname{Ker} A \cap D^{\infty}=\left\{\theta^{*}\right\}$, ce qui est absurde. La suite $\left(j\left(y_{n}\right)\right)_{n}$ est donc bornée. Il en est de même pour $\left(y_{n}\right)_{n}$. Or $A(C)+\varepsilon_{0} U^{*}$ est $w^{*}$-fermé et $\left(A(C)+\varepsilon_{0} U^{*}\right) \cap K=\emptyset$. D'après le théorème de Hahn-Banach [15], il existe $x$ non nul dans $X$ et $\eta \in \mathbb{R}$ tels que

$$
\sup _{t \in C}\langle x, A(t)\rangle+\varepsilon_{0}\|x\|<\eta<\inf _{t \in K}\langle x, t\rangle
$$

Il en résulte que $\sup _{t \in C}\langle x, A(t)\rangle=\sup _{t \in C}\left\langle A^{*}(x), t\right\rangle=I_{C}^{*}\left(A^{*}(x)\right) \in \mathbb{R}$ et $I_{C}^{*}\left(A^{*}(x)\right)<r:=\eta-\varepsilon_{0}\|x\|$, où $A^{*}: X \longrightarrow Y$ est l'opérateur adjoint de $A[16]$. Comme $C_{n} \stackrel{\tau_{s}^{*}}{\longrightarrow} C$, alors $0=I_{C_{n}}\left(y_{n}\right)>\left\langle A^{*}(x), y_{n}\right\rangle-r$, i.e. $\left\langle A^{*}(x), y_{n}\right\rangle<r$ pour tout $n$ assez grand. D'autre part, nous avons

$$
\begin{aligned}
\eta & <\left\langle x, A_{n}\left(y_{n}\right)+v_{n}\right\rangle \leq\left(\left\|v_{n}\right\|_{*}+\left\|A_{n}\left(y_{n}\right)-A\left(y_{n}\right)\right\|_{*}\right)\|x\|+\left\langle x, A\left(y_{n}\right)\right\rangle \\
& <r+\left(\left\|v_{n}\right\|_{*}+\left\|A_{n}\left(y_{n}\right)-A\left(y_{n}\right)\right\|_{*}\right)\|x\| .
\end{aligned}
$$

D’où, $\varepsilon_{0}<\left\|v_{n}\right\|_{*}+\left\|A_{n}\left(y_{n}\right)-A\left(y_{n}\right)\right\|_{*} \longrightarrow 0$, ce qui est absurde. Par conséquent $A_{n}\left(C_{n}\right) \stackrel{\tau_{s}^{*}}{\longrightarrow} A(C)$. Ce qui achève la preuve.

\section{VERSION FONCTIONNELLE DE LA STABILITÉ}

Dans cette section nous appliquerons les résultats de la section précédente aux épigraphes de fonctions convexes, ce qui nous permettera de déduire la stabilité au niveau fonctionnel. L'outil clé étant la notion d'inflocale compacité d'une fonction convexe.

Définition 4.1 [26]. Soit $X$ un espace topologique séparé. Une fonction $f \in \overline{\mathbb{R}}^{X}$ est dite inf-localement compacte si $f$ est sci et si pour tout $\rho \in \mathbb{R}$ l'ensemble $F_{\rho}:=\{x \in X / f(x) \leq \rho\}$ est localement compact.

Il est facile de remarquer que $f$ est inf-localement compacte si et seulement si son épigraphe epi $f$ est localement compact dans $X \times \mathbb{R}[26]$. Le résultat principal sur les fonctions inf-localement compactes est le suivant :

Proposition $4.2[26]$. Soit $X$ un e.l.c.s. et $f \in \Gamma(X)$. Alors $f$ est $w$-inf-localement compacte si et seulement si $f^{*}$ est $\tau^{*}$-quasi-continue.

Ce résultat généralise celui de Moreau [36] : une fonction $f \in \Gamma(X)$ est $w$-inf-compacte (i.e. $F_{\rho}$ est $w$-compact pour tout $\rho \in \mathbb{R}$ ) si et seulement si $f^{*}$ est $\tau^{*}$-continue en $\theta$.

Théorème 4.3. Soient $X$ un espace de Banach et $f_{n}, g_{n}, n \in I N, f, g$ des fonctions de $\Gamma(X)$ telles que $f_{n} \stackrel{\tau_{s}}{\longrightarrow} f$ et $g_{n} \stackrel{\tau_{s}}{\longrightarrow} g$. Supposons qu'il existe deux fonctions $p$ et $q$ de $\Gamma(X)$ telles que:

(1) $p \leq f_{n}$ et $q \leq g_{n}$ à partir d'un certain rang $n_{0}$;

(2) $q$ est $w$-inf-localement compacte;

(3) $\left\{x / p^{\infty}(x)+q^{\infty}(-x) \leq 0\right\}=\{\theta\}$.

Alors $f \# g, f_{n} \# g_{n}$ sont dans $\Gamma(X)$ pour $n$ assez grand, $f_{n} \# g_{n} \stackrel{\tau_{s}}{\longrightarrow} f \# g$ et l'inf dans ces fonctions est atteint.

Démonstration. L'hypothèse (1) est équivalente à epi $f_{n} \subset$ epi p et epi $g_{n} \subset$ epi q. L'hypothèse (2) est équivalente au fait que epi $q$ est $w \times \mid$.|-localement compact. D'autre part, il est facile de vérifier que l'hypothèse (3) est équivalente à la condition $(\text { epi } p)^{\infty} \cap-(e p i q)^{\infty}=\{\theta, 0\}$. Les hypothèses du théorème 3.11 sont donc satisfaites, par conséquent

$$
\text { epi } f_{n}+e p i g_{n} \stackrel{\tau_{s}}{\longrightarrow} \text { epif } f \text { epig }
$$


et ces ensembles sont fermés. Or epi $\left(f_{n} \# g_{n}\right)=e p i f_{n}+$ epi $g_{n}$ puisque epi $f_{n}+$ epi $g_{n} \subset$ epi $\left(f_{n} \# g_{n}\right) \subset$ cl $\left(\right.$ epi $\left.f_{n}+e p i g_{n}\right)=e p i f_{n}+e p i g_{n}$. De la même façon epi $(f \# g)=e p i f+e p i g$. Ainsi, $f_{n} \# g_{n} \stackrel{\tau_{s}}{\longrightarrow} f$ \# $g$ et ces fonctions sont convexes sci.

Montrons maintenant que $f_{n} \# g_{n}, f$ \# $g$ sont propres et l'inf dans ces fonctions est atteint : soient $x_{n} \in \operatorname{Dom} f_{n}$ et $y_{n} \in \operatorname{Dom} g_{n}$, alors

$$
\left(f_{n} \# g_{n}\right)\left(x_{n}+y_{n}\right) \leq f_{n}\left(x_{n}\right)+g_{n}\left(y_{n}\right)<+\infty .
$$

De la même façon $f \# g$ n'est pas identiquement égale à $+\infty$.

Pour montrer que $f_{n} \# g_{n}, f \# g$ ne prennent pas la valeur $-\infty$, il suffit de remarquer d'après (1) que $p \# q \leq \inf \left\{f_{n} \# g_{n}, f \# g\right\}$ et que $p \# q$ ne prend pas la valeur $-\infty$. En effet, l'hypothèse (3) et le lemme 2.1 impliquent que $p^{*}$ et $q^{*}$ sont unies dans la dualité $\left\langle X^{*}, X\right\rangle$. L'hypothèse (2) et la proposition 4.2 impliquent que $q^{*}$ est $\tau^{*}$-quasi-continue. Ainsi, Dom $p^{*} \cap D o m q^{*} \neq \emptyset$ d'après le lemme 6.6.I de [26]. Par conséquent $p$ \# $q$ ne prend pas la valeur $-\infty$. Maintenant les hypothèses (1)-(3) impliquent que $g_{n}$ et $g$ sont $w$-inf-localement compactes et

$$
\left\{x / f_{n}^{\infty}(x)+g_{n}^{\infty}(-x) \leq 0\right\}=\left\{x / f^{\infty}(x)+g^{\infty}(-x) \leq 0\right\}=\{\theta\} .
$$

Il en résulte en utilisant la proposition 6.4.I de [26] que $f_{n} \# g_{n}$ et $f \# g$ sont exactes, i.e. pour tout $x \in X$ il existe $z_{n} \in X$ et $z \in X$ tels que $\left(f_{n} \# g_{n}\right)(x)=f_{n}\left(z_{n}\right)+g_{n}\left(x-z_{n}\right)$ et $(f \# g)(x)=f(z)+g(x-z)$. Ceci achève la preuve du théorème.

Théorème 4.4. Soient $X$ un espace de Banach et $f_{n}, g_{n}, n \in \mathbb{I N}, f, g$ des fonctions de $\Gamma(X)$ telles que $f_{n} \stackrel{\tau_{s}}{\longrightarrow} f$ et $g_{n} \stackrel{\tau_{s}}{\longrightarrow} g$. Supposons qu'il existe deux fonctions $p$ et $q$ de $\Gamma(X)$ telles que :

(i) $f_{n} \leq p$ et $g_{n} \leq q$ à partir d'un certain rang $n_{0}$;

(ii) $q$ est $\|$.$\| -quasi-continue;$

(iii) $\cup_{\lambda \geq 0} \lambda(\operatorname{Dom} p-\operatorname{Dom} q)=X$.

Nous avons alors

(a) $f+g$ et $f_{n}+g_{n}$ sont propres et $f_{n}+g_{n} \stackrel{\tau_{s}}{\longrightarrow} f+g$.

(b) $G\left(\partial f_{n}+\partial g_{n}\right) \stackrel{P . K}{\longrightarrow} G(\partial f+\partial g)$.

Démonstration. (a) L'hypothèse (iii) implique que $\theta \in \operatorname{Dom} p-\operatorname{Dom} q[4]$ car $\cup_{\lambda \geq 0} \lambda$ (Dom $p-$ Dom q) est un espace vectoriel. Donc Dom $p \cap \operatorname{Dom} q \neq \emptyset$. Il en est de même pour Dom $f_{n} \cap \operatorname{Dom} g_{n}$ d'après l'hypothèse (i). Comme de plus $f_{n}+g_{n}>-\infty$ alors $f_{n}+g_{n}$ est propre. De la même façon $f+g$ est propre car $f+g>-\infty$ et d'après (i) $f \leq p$ et $g \leq q$.

Montrons maintenant que $f_{n}+g_{n} \stackrel{\tau_{s}}{\longrightarrow} f+g$.

L'hypothèse (i) est équivalente à $p^{*} \leq f_{n}^{*}$ et $q^{*} \leq g_{n}^{*}$ pour tout $n \geq n_{0}$. (ii) et la proposition 4.2 impliquent que $q^{*}$ est $w^{*}$-inf-localement compacte. Enfin $\left(\text { epi } p^{*}\right)^{\infty} \cap-\left(\text { epi } q^{*}\right)^{\infty}=\left\{\theta^{*}, 0\right\}$ d'après (iii) et le lemme 2.1. Comme de plus $f_{n}^{*} \stackrel{\tau_{s}^{*}}{\longrightarrow} f^{*}$ et $g_{n}^{*} \stackrel{\tau_{s}^{*}}{\longrightarrow} g^{*}[10]$ alors le théorème 3.14 appliqué à $C_{n}:=$ epi $f_{n}^{*}, C:=$ epi $f^{*}$, $D_{n}:=e p i g_{n}^{*}, D:=e p i g^{*}, C_{0}:=e p i p^{*}$ et $D_{0}:=e p i q^{*}$ implique que $C_{n}+D_{n}, C+D$ sont dans $\mathcal{C}\left(X^{*} \times \mathbb{R}\right)$ et $C_{n}+D_{n} \stackrel{\tau_{s}^{*}}{\longrightarrow} C+D$. Il en résulte que $f^{*} \# g^{*}$ et $f_{n}^{*} \# g_{n}^{*}$ sont dans $\Gamma\left(X^{*}\right)$ et $f_{n}^{*} \# g_{n}^{*} \stackrel{\tau_{s}^{*}}{\longrightarrow} f^{*} \# g^{*}$. Ce qui est équivalent par la $\tau_{s}-\tau_{s}^{*}$-bicontinuité de la transformation de Legendre-Fenchel [10] à

$$
f_{n}+g_{n}=\left(f_{n}^{*} \# g_{n}^{*}\right)^{*} \stackrel{\tau_{s}}{\longrightarrow}\left(f^{*} \# g^{*}\right)^{*}=f+g .
$$

(b) D'après [3], nous avons $G\left(\partial\left(f_{n}+g_{n}\right)\right) \stackrel{P . K}{\longrightarrow} G(\partial(f+g))$ car $f_{n}+g_{n} \stackrel{\tau_{s}}{\longrightarrow} f+g$. D'autre part les hypothèses (i) et (iii) impliquent que

$$
\cup_{\lambda \geq 0} \lambda\left(\operatorname{Dom} f_{n}-\operatorname{Dom} g_{n}\right)=\cup_{\lambda \geq 0} \lambda(\operatorname{Dom} f-\operatorname{Dom} g)=X,
$$

et d'après [4]

$$
\partial\left(f_{n}+g_{n}\right)=\partial f_{n}+\partial g_{n}, \partial(f+g)=\partial f+\partial g .
$$

D'où $G\left(\partial f_{n}+\partial g_{n}\right) \stackrel{P . K}{\longrightarrow} G(\partial f+\partial g)$ et le théorème est ainsi démontré. 
Donnons enfin, un exemple de suites de fonctions vérifiant les hypothèses du théorème 4.4 sans que $q$ soit continue :

Exemple 4.5. Soient $X$ un espace de Hilbert et $M_{1}$ et $M_{2}$ deux sous-espaces vectoriels fermés vérifiant $M_{1} \bigoplus M_{2}=X$ avec $\operatorname{dim} M_{1}<+\infty$ et $M_{1}$ non nul. Considérons les ensembles $C_{n}:=M_{1}+\frac{1}{n} U, D_{n}:=M_{2}+\frac{1}{n} U$ $(n \geq 1)$ et $C:=M_{1}, D:=M_{2}$ et les fonctions $f_{n}:=I_{C_{n}}, f:=I_{C}, g_{n}:=I_{D_{n}}$ et $g:=I_{D}$. On vérifie aisément que $f_{n} \stackrel{M}{\longrightarrow} f, g_{n} \stackrel{M}{\longrightarrow} g, f_{n} \leq p:=f, g_{n} \leq q:=g$ qui est quasi-continue mais non continue, et la condition (iii) du théorème 4.4 est aussi satisfaite.

Corollaire 4.6 [28]. Soient $X$ un espace de Banach et $f, g, f_{n}, g_{n}, n \in I N$ des fonctions de $\Gamma(X)$ telles que $f_{n} \stackrel{\tau_{s}}{\longrightarrow} f$ et $g_{n} \stackrel{\tau_{s}}{\longrightarrow} g$. Supposons qu'il existe un point $x_{0} \in$ Dom $f \cap$ Dom $g$ et $V$ un $\|$.$\| -voisinage de x_{0}$ tels que les fonctions $g_{n}$ soient uniformément majorées sur $V$. Alors $f_{n}+g_{n}, f+g$ sont propres et $f_{n}+g_{n} \stackrel{\tau_{s}}{\longrightarrow} f+g$.

Démonstration. Soit $B$ un $\|\cdot\|$-voisinage de $\theta$ tel que $V=x_{0}+B$ et soit $W$ un $\|$.\|-voisinage convexe fermé et équilibré de $\theta$ tel que $W+W \subseteq B$. Comme $f_{n} \stackrel{\tau_{s}}{\longrightarrow} f$ et $f\left(x_{0}\right)<+\infty$, il existe $\left(x_{n}\right)_{n} \subset X$ telle que $x_{n} \stackrel{\|\cdot\|}{\longrightarrow} x_{0}$ et $\sup _{n} f_{n}\left(x_{n}\right)<+\infty$. Posons $h_{n}:=f_{n}\left(.+x_{n}\right), e_{n}:=g_{n}\left(.+x_{n}\right), h:=f\left(.+x_{0}\right)$ et $e:=g\left(.+x_{0}\right)$. D'après le lemme 3.12, $h_{n} \stackrel{\tau_{s}}{\longrightarrow} h$ et $e_{n} \stackrel{\tau_{s}}{\longrightarrow} e$. De plus $h_{n} \leq p:=I_{\{\theta\}}+\alpha$ et $e_{n} \leq q:=I_{W}+\beta$, où $\alpha$ et $\beta$ sont deux réels tels que $h_{n}(\theta) \leq \alpha$ et $\sup _{x \in W} e_{n}(x) \leq \beta$ pour tout $n$. Les hypothèses (i), (ii) et (iii) du théorème 4.4 sont alors satisfaites, donc $h_{n}+e_{n}, h+e$ sont propres et $h_{n}+e_{n} \stackrel{\tau_{s}}{\longrightarrow} h+e$. D'où, $f+g, f_{n}+g_{n}$ sont propres et $f_{n}+g_{n} \stackrel{\tau_{s}}{\longrightarrow} f+g$ d'après le lemme 3.12. Ce qui achève la preuve.

Corollaire 4.7 [30]. Soient $f_{n}, g_{n}, n \in \mathbb{N}, f, g$ des fonctions de $\Gamma\left(\mathbb{R}^{p}\right)$ telles que $f_{n} \stackrel{e}{\longrightarrow} f, g_{n} \stackrel{e}{\longrightarrow} g$ et $f$ est finie continue en un point $x_{0}$ de Dom $g$. Alors $f_{n}+g_{n} \stackrel{e}{\longrightarrow} f+g$ et ces fonctions sont propres.

Démonstration. Soient $r>0$ et $M \in \mathbb{R}$ tels que $f(x)<M$ pour tout $x \in x_{0}+r U$. D'après [12,49],

$$
F_{n}:=\left\{x / f_{n}(x) \leq M\right\} \stackrel{P . K}{\longrightarrow} F:=\{x / f(x) \leq M\}
$$

et donc $\sup \left\{d\left(z, F_{n}\right) / z \in\left(x_{0}+r U\right) \cap F\right\}<\frac{r}{2}$ pour $n$ suffisamment grand d'après [6]. Ainsi $x_{0}+r U \subset F_{n}+\frac{r}{2} U$; et par [40], $x_{0}+\frac{r}{2} U \subset F_{n}$, i.e. les fonctions $f_{n}$ sont uniformément majorées sur $x_{0}+\frac{r}{2} U$. On conclut alors avec le corollaire 4.6.

Corollaire 4.8. Sous les hypothèses du théorème 4.4 nous avons $\max \left(f_{n}, g_{n}\right) \stackrel{\tau_{s}}{\longrightarrow} \max (f, g)$ et ces fonctions sont dans $\Gamma(X)$.

Si $X=\mathbb{R}^{p}$, il suffit que $f$ soit finie continue en un point de Dom g pour avoir la conclusion précédente.

Démonstration. Posons $h_{n}:=\max \left(f_{n}, g_{n}\right)$ et $h:=\max (f, g)$. Nous avons epi $h_{n}=$ epi $f_{n} \cap$ epi $g_{n}$, epi $h=$ epi $f \cap$ epi g. Maintenant il est facile de voir que $I_{\text {epi } f_{n}} \leq I_{\text {epi } p}, I_{\text {epi } g_{n}} \leq I_{\text {epi } q}$, epi $q$ est quasi-continue et $\bigcup_{\lambda \geq 0} \lambda($ epi p - epi q) $=X \times \mathbb{R}$. Toutes les hypothèses du théorème 4.4 sont satisfaites dans $X \times \mathbb{R}$, par conséquent

$$
I_{e p i h_{n}}=I_{e p i f_{n}}+I_{e p i g_{n}} \stackrel{\tau_{s}}{\longrightarrow} I_{e p i f}+I_{e p i g}=I_{e p i h}
$$

et ces fonctions sont dans $\Gamma(X \times \mathbb{R})$. Ainsi, $h_{n} \stackrel{\tau_{s}}{\longrightarrow} h$ et ces fonctions sont dans $\Gamma(X)$. Le reste découle immédiatement du corollaire 4.7 car epi $f_{n} \stackrel{P . K}{\longrightarrow}$ epi $f$, epi $g_{n} \stackrel{P . K}{\longrightarrow}$ epi $g$ et int $($ epi $f) \cap$ epi $g \neq \emptyset$.

Dans $[2,7]$, il est prouvé que la Mosco convergence est stable par l'addition de fonctions de $\Gamma(X)$ sous l'hypothèse suivante :

$$
(H): \exists s>0 \text { tel que } s U \subset \cup_{r \geq 0} \cap_{n \in \mathbb{N}}\left(\left\{f_{n} \leq r\right\} \cap r U-\left\{g_{n} \leq r\right\} \cap r U\right)
$$

Ce résultat ne peut pas être déduit du théorème 4.4. En effet, il suffit de prendre $X$ un espace de Hilbert de base orthonormale $\left(e_{n}\right)_{n>1}$ et $f_{n}:=g_{n}:=I_{C}=: f=: g$ avec $C:=\left\{x=\left(x_{1}, \ldots, x_{n}, \ldots\right) / x_{n} \geq 0, \forall n \geq 1\right\}$. Pourtant, nous avons démontré (dans un papier encore en préparation) que la slice convergence est stable par la somme de fonctions de $\Gamma(X)$ lorsque $(\mathrm{H})$ est satisfaite. 
Dans ce qui suit, nous montrons que la stabilité fonctionnelle est encore conservée par une classe d'opérateurs.

Pour une fonction $f: X \longrightarrow \overline{\mathbb{R}}$ et un opérateur $A: X \longrightarrow Y$, notons $R(A)$ l'image de $A$ et $A f$ la fonction définie par :

$$
y \in Y \longmapsto(A f)(y):=\inf \{f(x) / A(x)=y\} \in \overline{\mathbb{R}} .
$$

Si $B: Y \longrightarrow X$ est un autre opérateur, on définit la fonction notée $f \circ B$ par $: y \in Y \longmapsto(f \circ B)(y):=f(B(y))$.

Proposition 4.9. Soient $X$ et $Y$ deux espaces de Banach, $A_{n}, n \in I N, A: X \longrightarrow Y$ des opérateurs linéaires continus tels que $A_{n} \stackrel{U B}{\longrightarrow} A$ et $f_{n}, n \in I N, f$ des fonctions de $\Gamma(X)$ telles que $f_{n} \stackrel{\tau_{s}}{\longrightarrow} f$. Supposons qu'il existe une fonction $g$ de $\Gamma(\overrightarrow{X)} \pi$-inf-localement compacte telle que

$$
g \leq f_{n} \text { et } \operatorname{Ker} A \cap\left\{x \in X / g^{\infty}(x) \leq 0\right\}=\{\theta\} .
$$

Alors les fonctions $A_{n} f_{n}$, Af sont dans $\Gamma(Y), A_{n} f_{n} \stackrel{\tau_{s}}{\longrightarrow}$ Af et l'inf dans ces fonctions est atteint.

Démonstration. Considérons les opérateurs linéaires continus

$$
B_{n}, B: X \times \mathbb{R} \longrightarrow Y \times \mathbb{R}
$$

définis par : $B_{n}(x, \mu):=\left(A_{n}(x), \mu\right)$ et $B(x, \mu):=(A(x), \mu)$. Vérifions que les hypothèses du corollaire 3.5 sont satisfaites :

$-B_{n} \stackrel{U B}{\longrightarrow} B$ car $A_{n} \stackrel{U B}{\longrightarrow} A$;

- epi $\overrightarrow{f_{n}} \stackrel{\tau_{s}}{\longrightarrow}$ epi $f$ et epi $\vec{f}_{n} \subseteq$ epig qui est un convexe fermé $\pi \times \mid$.|-localement compact.

$$
\begin{aligned}
\operatorname{Ker} B \cap(\text { epig })^{\infty} & =\operatorname{Ker} B \cap e p i g^{\infty} \\
& =\left(\operatorname{Ker} A \cap\left\{x / g^{\infty}(x) \leq 0\right\}\right) \times\{0\} \\
& =\{(\theta, 0)\} .
\end{aligned}
$$

Ainsi, les ensembles $B_{n}\left(\right.$ epi $\left.f_{n}\right), B(e p i f)$ sont fermés et

$$
B_{n}\left(e p i f_{n}\right) \stackrel{\tau_{s}}{\longrightarrow} B(e p i f) .
$$

Or

$$
B_{n}\left(e p i f_{n}\right) \subseteq e p i\left(A_{n} f_{n}\right) \subseteq \operatorname{cl}\left(B_{n}\left(e p i f_{n}\right)\right)=B_{n}\left(e p i f_{n}\right)
$$

et

$$
B(\text { epif }) \subseteq e p i(A f) \subseteq c l(B(\text { epif }))=B(\text { epif }) .
$$

Il en résulte que les fonctions $A_{n} f_{n}, A f$ sont convexes sci et $A_{n} f_{n} \stackrel{\tau_{s}}{\longrightarrow} A f$.

Montrons que $A f, A_{n} f_{n}$ sont propres et que l'inf dans ces fonctions est atteint :

- soient $y:=A(x), x \in \operatorname{Dom} f$ et $y_{n}:=A_{n}\left(x_{n}\right), x_{n} \in \operatorname{Dom} f_{n}$. On a,

$$
A f(y) \leq f(x)<+\infty \text { et } A_{n} f_{n}\left(y_{n}\right) \leq f_{n}\left(x_{n}\right)<+\infty
$$

Donc $A f$ et $A_{n} f_{n}$ ne sont pas identiquement égales à $+\infty$;

- soient $y \in$ Dom $A f$ et $\beta$ un réel tel que $\beta>A f(y)$. Il existe $\left(x_{n}\right)_{n}$ telle que $f\left(x_{n}\right) \longrightarrow A f(y)$ et $A\left(x_{n}\right)=y$. L'ensemble $D:=\{x / f(x) \leq \beta\}$ est un convexe fermé non vide et $\pi$-localement compact. Quitte à faire une translation, on peut supposer sans nuire à la généralité que $\theta \in D$. Soit $j$ la jauge d'un voisinage $V$ de $\theta$ convexe fermé et équilibré tel que $V \cap D$ soit $\pi$ - compact. Comme $x_{n} \in D$ et $\operatorname{Ker} A \cap\left\{x / f^{\infty}(x) \leq 0\right\}=\{\theta\}$, alors selon un argument déjà utilisé, la suite $\left(j\left(x_{n}\right)\right)_{n}$ est bornée et $x_{n} \stackrel{w}{\longrightarrow} x \in D$ pour une sous-suite. Par suite, $f(x) \leq \underline{\lim } f\left(x_{n}\right)=A f(y)$ et $A(x)=y$. Ainsi $A f(y)=f(x)>-\infty$ et $A f$ est propre;

- un raisonnement analogue au précédent montre que pour $n$ suffisamment grand, l'inf dans $A_{n} f_{n}$ est atteint et que cette fonction est propre car la condition $\operatorname{Ker} A_{n} \cap\left\{x / f_{n}^{\infty}(x) \leq 0\right\}=\{\theta\}$ est encore satisfaite à partir d'un certain rang puisque $\operatorname{Ker} B_{n} \cap\left(\text { epi } f_{n}\right)^{\infty}=\{(\theta, 0)\}$. 
Proposition 4.10. Soient $X$ et $Y$ deux espaces de Banach, $A_{n}, n \in I N, A: Y^{*} \longrightarrow X^{*}$ des opérateurs linéaires $\left(\|\cdot\|_{*},\|\cdot\|_{*}\right)$ et $\left(w^{*}, w^{*}\right)$-continus tels que $A_{n} \underset{U B}{\longrightarrow} A$ et $f_{n}, n \in I N, f$ des fonctions de $\Gamma\left(Y^{*}\right)$ telles que $f_{n} \stackrel{\tau_{s}^{*}}{\longrightarrow} f$. Supposons qu'il existe une fonction $g$ de $\Gamma\left(Y^{*}\right) \pi^{*}$-inf-localement compacte telle que

$$
g \leq f_{n} \text { et } \operatorname{Ker} A \cap\left\{y \in X / g^{\infty}(y) \leq 0\right\}=\left\{\theta^{*}\right\} .
$$

Alors les fonctions $A_{n} f_{n}$, Af sont dans $\Gamma\left(X^{*}\right), A_{n} f_{n} \stackrel{\tau_{s}^{*}}{\longrightarrow}$ Af et l'inf dans ces fonctions est atteint.

Démonstration. La slice convergence duale des fonctions $A_{n} f_{n}$ vers $A f$ ainsi que leur $w^{*}$-sci se démontrent exactement comme dans la preuve de la proposition 4.9 moyennant le théorème 3.15 appliqué aux ensembles $B_{n}\left(\right.$ epi $\left.f_{n}\right), B\left(\right.$ epif) avec $B_{n}, B: Y^{*} \times \mathbb{R} \longrightarrow X^{*} \times \mathbb{R}$ les opérateurs linéaires continus définis comme précédemment. Pour justifier la propreté des fonctions $A f$ et $A_{n} f_{n}$, nous allons raisonner en terme de valeurs d'adhérence et non en terme de sous-suites convergentes car $Y$ n'est pas en général séparable. En effet, soient $y \in D o m$ Af $\beta \in \mathbb{R}$ tel que $\beta>A f(y)$ et $\left(v_{n}\right)_{n}$ une suite telle que $f\left(v_{n}\right) \longrightarrow A f(y)$ et $A\left(v_{n}\right)=y$. Soit $D:=\left\{z \in Y^{*} / f(z) \leq \beta\right\}$. Il est clair que $D$ est un convexe non vide $w^{*}$-fermé et $\pi^{*}$-localement compact; et comme dans la preuve de la proposition 4.9 , nous avons $\left(j\left(v_{n}\right)\right)_{n}$ est bornée et la suite $\left(v_{n}\right)_{n}$ admet une valeur d'adhérence $v$ pour la topologie $w^{*}$. Comme $A$ est $\left(w^{*}, w^{*}\right)$-continu alors $A(v)$ est une valeur d'adhérence de $\left(A\left(v_{n}\right)\right)_{n}$, par suite $A(v)=y$. Montrons maintenant que $f(v)=A f(y)$. Par définition nous avons $A f(y) \leq f(v)$. Si $A f(y)<f(v)$, considérons alors $\alpha \in \mathbb{R}$ tel que $A f(y)<\alpha<f(v)$. Comme $f$ est $w^{*}$-sci, il existe un $w^{*}$-voisinage $W$ de $v$ tel que $\alpha<f(z)$ pour tout $z \in W$. D'autre part, nous avons $f\left(v_{n}\right)<\alpha$ pour tout $n \geq N$ car $f\left(v_{n}\right) \longrightarrow A f(y)$. Mais puisque $v$ est une valeur d'adhérence de $\left(v_{n}\right)_{n}$, il existe une infinité d'indices $n \geq N$ tels que $v_{n} \in W$. Si $n$ est un tel indice alors $f\left(v_{n}\right)<\alpha<f\left(v_{n}\right)$, ce qui est absurde. Donc $A f(y)=f(v)>-\infty$ et $A f$ est propre. La propreté de $A_{n} f_{n}$ à partir d'un certain rang se démontre de la même façon. Ceci achève la preuve de la proposition.

Théorème 4.11. Soient $X$ et $Y$ deux espaces de Banach, $A_{n}, n \in I N, A: X \longrightarrow Y$ des opérateurs linéaires continus tels que $A_{n} \stackrel{U B}{\longrightarrow}$ A et $f_{n}, n \in \mathbb{I}, f$ des fonctions de $\Gamma(Y)$ telles que $f_{n} \stackrel{\tau_{s}}{\longrightarrow} f$. Supposons qu'il existe une fonction $g$ de $\Gamma(Y)\|$.$\| -quasi-continue et vérifiant:$

(1) $f_{n} \leq g, \forall n \geq \grave{a}$ un certain $N$;

(2) $\cup_{\lambda \geq 0} \lambda(R(A)-D o m g)=Y$.

Alors les fonctions $f_{n} \circ A_{n}, f \circ A$ sont dans $\Gamma(X)$ pour $n$ suffisamment grand et $f_{n} \circ A_{n} \stackrel{\tau_{s}}{\longrightarrow} f \circ A$.

Pour la preuve de ce théorème, nous utiliserons le lemme suivant :

Lemme 4.12 [34]. Soient $X, Y$ deux espaces de Banach, $A: X \longrightarrow Y$ un opérateur linéaire continu et $g$ une fonction quasi-continue de $\Gamma(Y)$. Les propriétés suivantes sont équivalentes :

1) $\operatorname{Ker}^{*} \cap\left\{z / g^{* \infty}(z) \leq 0\right\}=\left\{\theta^{*}\right\}$;

2) $R(A)^{\perp} \cap\left\{z / g^{* \infty}(z) \leq 0\right\}=\left\{\theta^{*}\right\}$;

3) $\cup_{\lambda \geq 0} \lambda(\overline{R(A)}-D o m g)=Y$;

4) $\cup_{\lambda \geq 0} \lambda(R(A)-D o m g)=Y$.

Démonstration du théorème 4.11. D'après le lemme précédent, (2) est équivalente à $\operatorname{Ker} A^{*} \cap\left\{z / g^{* \infty}(z) \leq 0\right\}=$ $\left\{\theta^{*}\right\}$,où $A^{*}: Y^{*} \longrightarrow X^{*}$ désigne l'opérateur adjoint de $A$ défini par $\left\langle x, A^{*}\left(y^{*}\right)\right\rangle=\left\langle A(x), y^{*}\right\rangle \forall x \in X \quad \forall y^{*} \in$ $Y^{*}$. D'autre part, $g^{*}$ est $w^{*}$-inf-localement compacte par la proposition 4.2 et $g^{*} \leq f_{n}^{*}$, puis $A_{n}^{*} \underset{U B}{\longrightarrow} A^{*}$ et ces opérateurs sont $\left(\|\cdot\|_{*},\|\cdot\|_{*}\right)$ et $\left(w^{*}, w^{*}\right)$-continus. Il en résulte par application de la proposition 4.10 que $A_{n}^{*} f_{n}^{*}, A^{*} f^{*}$ sont dans $\Gamma\left(X^{*}\right)$ et $A_{n}^{*} f_{n}^{*} \stackrel{\tau_{s}^{*}}{\longrightarrow} A^{*} f^{*}$; et par la $\tau_{s}-\tau_{s}^{*}$-bicontinuité de la transformation de LegendreFenchel [10]

et ces fonctions sont dans $\Gamma(X)$ pour $n$ suffisamment grand. 
Corollaire 4.13 [30]. Soient $A_{n}, n \in I N, A: \mathbb{R}^{p} \longrightarrow \mathbb{R}^{q}$ des opérateurs linéaires tels que $A_{n} \longrightarrow A$ et $f_{n}, n \in I N, f$ sont des fonctions de $\Gamma\left(\mathbb{R}^{q}\right)$ telles que $f_{n} \stackrel{e}{\longrightarrow} f$ et $f$ est finie continue en un point $z_{0}$ de $R(A)$. Alors $f_{n} \circ A_{n}, f \circ A$ sont dans $\Gamma\left(\mathbb{R}^{p}\right)$ pour $n$ suffisamment grand et $f_{n} \circ A_{n} \stackrel{e}{\longrightarrow} f \circ A$.

Démonstration. Selon un argument déjà utilisé, il existe une constante $M$ et $r>0$ tels que $f_{n} \leq g:=I_{z_{0}+r U}+M$, $\forall n \geq$ à un certain $N$. D'autre part la condition $\operatorname{int}(\operatorname{Dom} f) \cap R(A) \neq \emptyset$ implique que $\theta \in \operatorname{int}(R(A)-\operatorname{Dom} f)$ et donc $\cup_{\lambda \geq 0} \lambda(R(A)-D o m f)=Y$. Les hypothèses du théorème 4.11 sont donc satisfaites. Par suite, $f_{n} \circ$ $A_{n}, f \circ A$ sont dans $\Gamma\left(\mathbb{R}^{p}\right)$ pour $n$ suffisamment grand et $f_{n} \circ A_{n} \stackrel{e}{\longrightarrow} f \circ A$.

\section{Application À la CONVERGence D'un schema de DUAlité en optimisation CONVEXE}

Dans cette section, nous appliquons les résultats de stabilité des sections 3 et 4 à la convergence d'un schéma de dualité en optimisation convexe dans un cadre non nécessairement réflexif. Plus précisémment, nous donnerons des conditions de qualification naturelles assurant la convergence d'une suite de problèmes d'optimisation primaux (resp. duaux) vers le problème limite primal (resp. dual). L'outil de base étant la slice convergence et les fonctions de perturbations présentées ici ont une forme particulière fort intéressante dans les applications et qui s'adapte parfaitement avec la convergence précitée du point de vue stabilité (voir les lems. 5.3 et 5.4).

Hypothèse 5.1. Soient $X$ et $Y$ deux espaces de Banach avec $Y$ séparable, $f, f_{n}$ des fonctions de $\Gamma(X)$ et $g, g_{n}$ des fonctions de $\Gamma(Y)$ telles que $f_{n} \stackrel{\tau_{s}}{\longrightarrow} f$ et $g_{n} \stackrel{\tau_{s}}{\longrightarrow} g$. Considérons les fonctions de perturbations verticales définies par :

$$
\Phi_{n}(x, y):=f_{n}(x)+g_{n}\left(\Lambda_{n}(x)-y\right), \quad \Phi(x, y):=f(x)+g(\Lambda(x)-y)
$$

où $\Lambda, \Lambda_{n}: X \longrightarrow Y$ sont des opérateurs linéaires continus tels que $\Lambda_{n} \stackrel{U B}{\longrightarrow} \Lambda$. La présentation de ces fonctions sous cette forme a un intérêt fort intéressant dans les applications [21]. Dans la suite nous considérons les problèmes de minimisations primaux :

$$
\left(\mathcal{P}_{n}\right): \inf _{x \in X} \Phi_{n}(x, \theta), \quad(\mathcal{P}): \inf _{x \in X} \Phi(x, \theta)
$$

Les problèmes duaux associés sont notés respectivement :

$$
\left(\mathcal{P}_{n}^{*}\right): \sup _{y^{*} \in Y^{*}}\left\{-\Phi_{n}^{*}\left(\theta^{*}, y^{*}\right)\right\}, \quad\left(\mathcal{P}^{*}\right): \sup _{y^{*} \in Y^{*}}\left\{-\Phi^{*}\left(\theta^{*}, y^{*}\right)\right\}
$$

où $\Phi_{n}^{*}$ (resp. $\left.\Phi^{*}\right)$ est la transformée de Legendre-Fenchel de $\Phi_{n}$ (resp. $\left.\Phi\right)$ dans la dualité $\left\langle X \times Y, X^{*} \times Y^{*}\right\rangle$. Il est classique de vérifier $[21,31]$ que :

$$
\begin{aligned}
& \Phi_{n}^{*}\left(\theta^{*}, y^{*}\right)=f_{n}^{*}\left(\Lambda_{n}^{*}\left(y^{*}\right)\right)+g_{n}^{*}\left(-y^{*}\right)=: h_{n}^{*}\left(y^{*}\right), \\
& \Phi^{*}\left(\theta^{*}, y^{*}\right)=f^{*}\left(\Lambda^{*}\left(y^{*}\right)\right)+g^{*}\left(-y^{*}\right)=: h^{*}\left(y^{*}\right),
\end{aligned}
$$

où $h_{n}^{*}\left(\right.$ resp. $\left.h^{*}\right)$ est la transformée de Legendre-Fenchel de la fonction marginale $h_{n}(y):=\inf _{x \in X} \Phi_{n}(x, y)$ (resp. $\left.h(y):=\inf _{x \in X} \Phi(x, y)\right)$ et $\Lambda_{n}^{*}, \Lambda^{*}$ sont les opérateurs adjoints de $\Lambda_{n}$ et $\Lambda$.

Nous avons toujours $\sup \mathcal{P}^{*} \leq \inf \mathcal{P}$ et l'égalité a lieu si $h$ est sci en $\theta$. Pour plus de détails sur la dualité et ses applications le lecteur pourra consulter [21,29].

Le problème que nous présentons ici est le suivant : sous quelles conditions les problèmes $(\mathcal{P})$ et $\left(\mathcal{P}^{*}\right)$ admettent-ils des solutions que l'on peut approcher par des solutions des problèmes « approchés $\left(\mathcal{P}_{n}\right)$ et $\left(\mathcal{P}_{n}^{*}\right)$ ? 
Nous répondons à cette question dans le théorème suivant :

Théorème 5.2. On suppose en plus des données et des hypothèses de 5.1 que les conditions suivantes soient satisfaites :

1) il existe une fonction $p \in \Gamma(X \times Y)$ inf-localement compacte pour une topologie d'e.l.c.s. compatible avec la dualité $\left\langle X \times Y, X^{*} \times Y^{*}\right\rangle$ telle que $p(x, y) \leq \Phi_{n}(x, y)$ pour tout $(x, y) \in X \times Y$ et tout $n$ assez grand;

2) $p(., \theta)$ est $w$-inf-compacte;

3) les $h_{n}$ sont uniformément majorées sur un voisinage de $\theta$. Alors :

a) $\left(\mathcal{P}_{n}\right)$ et $(\mathcal{P})$ admettent des solutions ainsi que les problèmes $\left(\mathcal{P}_{n}^{*}\right)$ et $\left(\mathcal{P}^{*}\right)$, de plus $\inf \mathcal{P}_{n}=\sup \mathcal{P}_{n}^{*}$ et $\inf \mathcal{P}=\sup \mathcal{P}^{*}$

b) $\inf \mathcal{P}_{n} \longrightarrow \inf \mathcal{P}$; et si $\left(x_{n}\right)_{n}$ et $\left(y_{n}^{*}\right)_{n}$ sont deux suites telles que

$$
\Phi_{n}\left(x_{n}, \theta\right) \leq \inf \mathcal{P}_{n}+\varepsilon_{n} \text { et } \sup \mathcal{P}_{n}^{*}-\varepsilon_{n} \leq-\Phi_{n}^{*}\left(\theta^{*}, y_{n}^{*}\right) \text { avec } \varepsilon_{n} \downarrow 0,
$$

alors il existe une sous-suite $\left(n_{k}\right)_{k}$ telle que $\left(x_{n_{k}}, y_{n_{k}}^{*}\right) \stackrel{w \times w^{*}}{\longrightarrow}\left(\bar{x}, \overline{y^{*}}\right)$ avec $\bar{x}$ solution de $(\mathcal{P})$ et $\overline{y^{*}}$ solution de $\left(\mathcal{P}^{*}\right)$.

Pour la preuve de ce théorème nous utiliserons les deux lemmes suivants :

Lemme 5.3. Sous les hypothèses de 5.1, $\Phi_{n} \stackrel{\tau_{s}}{\longrightarrow} \Phi$ et ces fonctions sont dans $\Gamma(X \times Y)$.

Démonstration. Il est clair que $\Phi_{n}, \Phi$ sont convexes sci et ne prennent jamais la valeur $-\infty$. De plus, si $x \in \operatorname{Dom} f$ et $z \in$ Dom $g$ alors

$$
\Phi(x, \Lambda(x)-z)=f(x)+g(z)<+\infty,
$$

donc $\Phi$ n'est pas identiquement égale à $+\infty$. De la même façon $D o m \Phi_{n} \neq \emptyset$, et donc $\Phi_{n}$, $\Phi$ sont dans $\Gamma(X \times Y)$. Montrons que $\Phi_{n} \stackrel{\tau_{s}}{\longrightarrow} \Phi$.

(i) Soit $(x, y) \in X \times Y$. Puisque $f_{n} \stackrel{\tau_{s}}{\longrightarrow} f$ et $g_{n} \stackrel{\tau_{s}}{\longrightarrow} g$, il existe $\left(\left(x_{n}, z_{n}\right)\right)_{n}$ telle que $x_{n} \stackrel{\|\cdot\|}{\longrightarrow} x, z_{n} \stackrel{\|\cdot\|}{\longrightarrow} \Lambda(x)-y$, $f_{n}\left(x_{n}\right) \longrightarrow f(x)$ et $g_{n}\left(z_{n}\right) \longrightarrow g(\Lambda(x)-y)$. En posant $y_{n}:=\Lambda_{n}\left(x_{n}\right)-z_{n}$, nous avons $\left\|\left(x_{n}, y_{n}\right)-(x, y)\right\|_{X \times Y} \longrightarrow 0$ et

$$
\Phi_{n}\left(x_{n}, y_{n}\right)=f_{n}\left(x_{n}\right)+g_{n}\left(z_{n}\right) \longrightarrow f(x)+g(\Lambda(x)-y)=\Phi(x, y) .
$$

(ii) Soit $\left(x^{*}, y^{*}\right) \in X^{*} \times Y^{*}$. Comme $f_{n}^{*} \stackrel{\tau_{s}^{*}}{\longrightarrow} f^{*}$ et $g_{n}^{*} \stackrel{\tau_{s}^{*}}{\longrightarrow} g^{*}$, il existe $\left(\left(t_{n}^{*}, z_{n}^{*}\right)\right)_{n}$ telle que $t_{n}^{*} \stackrel{\|\cdot\|_{*}}{\longrightarrow} \Lambda^{*}\left(y^{*}\right)+x^{*}$, $z_{n}^{*} \stackrel{\|\cdot\|_{*}}{\longrightarrow}-y^{*}, g_{n}^{*}\left(z_{n}^{*}\right) \longrightarrow g^{*}\left(-y^{*}\right)$ et $f_{n}^{*}\left(t_{n}^{*}\right) \longrightarrow f^{*}\left(\Lambda^{*}\left(y^{*}\right)+x^{*}\right)$. En posant $x_{n}^{*}:=t_{n}^{*}-\Lambda_{n}^{*}\left(y_{n}^{*}\right), y_{n}^{*}:=-z_{n}^{*}$, il est clair que $\left\|\left(x_{n}^{*}, y_{n}^{*}\right)-\left(x^{*}, y^{*}\right)\right\|_{X^{*} \times Y^{*}} \longrightarrow 0$,

$$
\Phi_{n}^{*}\left(x_{n}^{*}, y_{n}^{*}\right)=f_{n}^{*}\left(\Lambda_{n}^{*}\left(y_{n}^{*}\right)+x_{n}^{*}\right)+g_{n}^{*}\left(-y_{n}^{*}\right)=f_{n}^{*}\left(t_{n}^{*}\right)+g_{n}^{*}\left(z_{n}^{*}\right)
$$

et

$$
\Phi^{*}\left(x^{*}, y^{*}\right)=f^{*}\left(\Lambda^{*}\left(y^{*}\right)+x^{*}\right)+g^{*}\left(-y^{*}\right) .
$$

Par suite $\Phi_{n}^{*}\left(x_{n}^{*}, y_{n}^{*}\right) \longrightarrow \Phi^{*}\left(x^{*}, y^{*}\right)$. Ce qui achève la preuve.

Lemme 5.4. Sous les hypothèses du théorème $5.2, h, h_{n} \in \Gamma(Y)$ pour tout $n$ suffisamment grand et $h_{n} \stackrel{\tau_{s}}{\longrightarrow} h$.

Démonstration. Soit $A:(x, y, \alpha) \in X \times Y \times \mathbb{R} \longrightarrow(y, \alpha) \in Y \times \mathbb{R}$.

Définissons les ensembles $C_{n}:=e p i \Phi_{n}$ et $C:=e p i \Phi$. D'après le lemme 5.3, nous avons $C_{n} \stackrel{\tau_{s}}{\longrightarrow} C$ et $C_{n} \subset D:=$ epi p qui est localement compact pour une topologie d'e.l.c.s. compatible avec la dualité $\left\langle X \times \vec{Y} \times \mathbb{R}, X^{*} \times Y^{*} \times \mathbb{R}\right\rangle$ d'après l'hypothèse 1 du théorème 5.2. D'autre part,

$$
(x, y, \alpha) \in \operatorname{Ker} A \cap(\text { epip })^{\infty} \Longleftrightarrow(y, \alpha)=(\theta, 0) \text { et }(x, \theta, 0) \in \text { epi } p^{\infty} .
$$


Cette dernière condition est équivalente à $p^{\infty}(x, \theta) \leq 0$. Soit $n$ suffisamment grand de sorte que $h_{n}(\theta)<+\infty$. Il existe alors, d'après les hypothèses 1 et $3, x_{0} \in X$ tel que $p\left(x_{0}, \theta\right)<+\infty$ et donc

$$
p^{\infty}(x, \theta)=\sup _{\lambda>0} \frac{p\left(x_{0}+\lambda x, \theta\right)-p\left(x_{0}, \theta\right)}{\lambda}
$$

Par suite, $p^{\infty}(x, \theta) \leq 0$ si et seulement si $p\left(x_{0}+\lambda x, \theta\right) \leq p\left(x_{0}, \theta\right)$ pour tout $\lambda>0$; mais d'après l'hypothèse 2 , ceci ne peut avoir lieu que si $x=\theta$. Ainsi $\operatorname{Ker} A \cap D^{\infty}=\{(\theta, \theta, 0)\}$. Les ensembles $A(C), A\left(C_{n}\right)$ sont alors fermés pour $n$ suffisamment grand et $A\left(C_{n}\right) \stackrel{\tau_{s}}{\longrightarrow} A(C)$ d'après le corollaire 3.5. Or, $A\left(C_{n}\right) \subset \operatorname{epi} h_{n} \subset \operatorname{cl}\left(A\left(C_{n}\right)\right)=$ $A\left(C_{n}\right)$, i.e. epi $h_{n}=A\left(C_{n}\right)$, de même epi $h=A(C)$. Donc $h$ et $h_{n}$ sont convexes sci et epi $h_{n} \stackrel{\tau_{s}}{\longrightarrow}$ epi $h$. On en déduit en utilisant l'hypothèse 3 que $h$ est majorée sur un voisinage de $\theta$. Si $h$ prend la valeur $-\infty$ en un point de $Y$, nécessairement $h(\theta)=-\infty$. Il existe alors une suite $\left(x_{k}\right)_{k}$ telle que $\Phi\left(x_{k}, \theta\right)<-k$ pour tout $k$. Mais d'après le lemme 5.3 et l'hypothèse 1 du théorème 5.2 nous avons $p\left(x_{k}, \theta\right) \leq \Phi\left(x_{k}, \theta\right) \leq-k \leq 0$. Or d'après l'hypothèse $2, p(., \theta)$ est $w$-inf-compacte et $x_{k} \stackrel{w}{\longrightarrow} \bar{x}$ pour une sous-suite. Ainsi $p(\bar{x}, \theta) \leq \underline{\lim } p\left(x_{k}, \theta\right)=-\infty$, ce qui est absurde car $p$ est est propre. Donc $h$ ne prend pas la valeur $-\infty$, par suite $h \in \Gamma(Y)$. De la même façon, $h_{n} \in \Gamma(Y)$ pour tout $n$ suffisamment grand, et $h_{n} \stackrel{\tau_{s}}{\longrightarrow} h$ puisque epi $h_{n} \stackrel{\tau_{s}}{\longrightarrow}$ epi $h$. Ceci achève la preuve du lemme.

Démonstration du théorème 5.2. a) Les hypothèses 1,2 et 3 impliquent que $\Phi_{n}(., \theta)$ est une fonction propre $w$-inf-compacte, par suite $\left(\mathcal{P}_{n}\right)$ admet au moins une solution. L'hypothèse 1 et le lemme 5.3 impliquent que $p \leq \Phi$ et d'après l'hypothèse $2,(\mathcal{P})$ admet au moins une solution. D'autre part les fonctions $h_{n}^{*}$, $h^{*}$ sont $w^{*}$-inf-compactes car $h_{n}, h$ sont continues en $\theta[29,36]$. Ainsi, $\left(\mathcal{P}_{n}^{*}\right)$ et $\left(\mathcal{P}^{*}\right)$ admettent au moins une solution et

$$
\inf \mathcal{P}_{n}=\sup \mathcal{P}_{n}^{*}, \inf \mathcal{P}=\sup \mathcal{P}^{*}
$$

b) Les fonctions $h_{n}$ étant uniformément majorées sur un $\|$.\|-voisinage de $\theta$, sont donc $\|$. $\|$-équi-sci en ce point. D'autre part par le lemme 5.4, nous avons $h_{n} \stackrel{\tau_{s}}{\longrightarrow} h$. Ainsi $h_{n}(\theta) \longrightarrow h(\theta)$ d'après [1, 19], i.e. $\inf \mathcal{P}_{n} \longrightarrow \inf \mathcal{P}$. Soit maintenant $\left(x_{n}\right)_{n},\left(y_{n}^{*}\right)_{n}$ et $\left(\varepsilon_{n}\right)_{n}$ des suites telles que $\varepsilon_{n} \downarrow 0$ et

$$
\Phi_{n}\left(x_{n}, \theta\right) \leq \inf \mathcal{P}_{n}+\varepsilon_{n}, \quad \sup \mathcal{P}_{n}^{*}-\varepsilon_{n} \leq-\Phi_{n}^{*}\left(\theta^{*}, y_{n}^{*}\right)
$$

D'après l'hypothèse 3 il existe $M \in \mathbb{R}$ tel que $h_{n}(\theta)+\varepsilon_{n} \leq M$, et en vertu de l'hypothèse $1 x_{n} \in\{x / p(x, \theta) \leq M\}$ qui est $w$-compact. Donc $x_{n} \stackrel{w}{\longrightarrow} \bar{x}$ pour une sous-suite; et par le lemme $5.3 \Phi(\bar{x}, \theta) \leq \underline{\lim } \Phi_{n}\left(x_{n}, \theta\right) \leq \underline{\lim } h_{n}(\theta)=$ $h(\theta)$, i.e. $h(\theta)=\Phi(\bar{x}, \theta)$ et $\bar{x}$ est une solution de $(\mathcal{P})$. Maintenant puisque les fonctions $h_{n}$ sont uniformément majorées sur un voisinage de $\theta$, il existe $\alpha \in \mathbb{R}$ et $r>0$ tels que $h_{n} \leq I_{r U}+\alpha$, i.e. $h_{n}^{*} \geq r\|.\|_{*}-\alpha$. La suite $\left(y_{n}^{*}\right)_{n}$ vérifie alors $r\left\|y_{n}^{*}\right\|_{*}-\alpha-\varepsilon_{n} \leq h_{n}^{*}\left(y_{n}^{*}\right)-\varepsilon_{n} \leq \inf _{z^{*}} h_{n}^{*}\left(z^{*}\right)=-h_{n}(\theta)$. Comme $h_{n}(\theta) \longrightarrow h(\theta) \in \mathbb{R},\left(y_{n}^{*}\right)_{n}$ est bornée et $y_{n}^{*} \stackrel{w^{*}}{\longrightarrow} \overline{y^{*}}$ pour une sous-suite car $Y$ est séparable. Mais vu que $h_{n}^{*} \stackrel{\tau_{s}^{*}}{\longrightarrow} h^{*}$, on obtient [10], p. 278, $h^{*}\left(\overline{y^{*}}\right) \leq \underline{\lim } h_{n}^{*}\left(y_{n}^{*}\right) \leq \underline{\lim }\left(-\sup \mathcal{P}_{n}^{*}\right)=-\varlimsup \lim \left(\sup \mathcal{P}_{n}^{*}\right)=-\sup \mathcal{P}^{*}$, i.e. $\overline{y^{*}}$ est solution de $\left(\mathcal{P}^{*}\right)$. Ce qui achève la preuve du théorème.

En dimension finie on obtient les mêmes conclusions du théorème 5.2 avec des hypothèses plus faibles.

Théorème 5.5. Supposons que les hypothèses de 5.1 soient satisfaites avec $X:=\mathbb{R}^{p}$ et $Y:=\mathbb{R}^{q}$. Supposons en outre que :

1) $\lim _{\|x\| \longrightarrow+\infty} \Phi(x, \theta)=+\infty$;

2) $h$ est finie et continue en $\theta$.

Alors les conclusions du théorème 5.2 restent valables et les convergences des solutions des problèmes $\left(\mathcal{P}_{n}\right)$ et $\left(\mathcal{P}_{n}^{*}\right)$ ont lieu au sens des normes.

Les éléments clés dans la preuve de ce théorème sont d'une part l'épi-convergence des fonctions marginales $h_{n}$ et d'autre part une notion d'uniforme inf-compacité des fonctions $\Phi_{n}(., \theta)$. 
Lemme 5.6 [48]. Soient $\left.\left.f_{n}, n \in \mathbb{I N}: \mathbb{R}^{p} \longrightarrow\right]-\infty,+\infty\right]$ des fonctions sci telles que pour tout $n \in \mathbb{N}$ et tout $\alpha \in \mathbb{R}$ l'ensemble $\left\{x / f_{n}(x) \leq \alpha\right\}$ est connexe. Supposons que la fonction notée

$$
x \longrightarrow \underline{\lim e} f_{n}(x):=\inf \left\{\underline{\lim } f_{n}\left(x_{n}\right) / x_{n} \stackrel{\|\cdot\|}{\longrightarrow} x\right\}
$$

soit inf-compacte. Alors les $f_{n}$ sont uniformément inf-compactes :

$$
\forall \alpha \in \mathbb{R}, \exists N_{\alpha} \in \mathbb{I N} \text { et } K_{\alpha} \text { compact tels que } \forall n \geq N_{\alpha} \quad\left\{x / f_{n}(x) \leq \alpha\right\} \subset K_{\alpha} .
$$

Lemme 5.7. Sous les hypothèses du théorème $5.5, h_{n}$ et $h$ sont dans $\Gamma\left(\mathbb{R}^{q}\right)$ pour $n$ suffisamment grand et $h_{n} \stackrel{e}{\longrightarrow} h$.

Démonstration. Soient $A, C$ et $C_{n}$ l'opérateur et les ensembles du lemme 5.4. Nous avons $C_{n} \stackrel{P . K}{\longrightarrow} C$ puisque en dimension finie, la slice convergence d'ensembles convexes fermés est identique à la convergence au sens de Painlevé-Kuratowski. Pour montrer que $A\left(C_{n}\right) \stackrel{P . K}{\longrightarrow} A(C)$, il suffit d'après [30] de vérifier que $\operatorname{Ker} A \cap C^{\infty}=$ $\{(\theta, \theta, 0)\}$ : en effet,

$$
(x, y, \alpha) \in \operatorname{Ker} A \cap C^{\infty} \Longleftrightarrow y=\theta, \alpha=0 \text { et } \Phi^{\infty}(x, \theta) \leq 0 .
$$

Comme $h$ est finie en $\theta$, il existe $x_{0} \in X$ tel que $\Phi\left(x_{0}, \theta\right) \in \mathbb{R}$. D'où,

$$
\Phi^{\infty}(x, \theta) \leq 0 \Longleftrightarrow \Phi\left(x_{0}+\lambda x, \theta\right) \leq \Phi\left(x_{0}, \theta\right), \forall \lambda>0,
$$

ce qui ne peut avoir lieu d'après l'hypothèse 1 que si $x=\theta$. Ainsi, $A\left(C_{n}\right) \stackrel{P . K}{\longrightarrow} A(C)$ et ces ensembles sont convexes fermés pour tout $n$ suffisamment grand. On en déduit comme dans la preuve du lemme 5.4 que epi $h_{n} \stackrel{P . K}{\longrightarrow}$ epi $h$ et ces ensembles sont dans $\mathcal{C}\left(\mathbb{R}^{q} \times \mathbb{R}\right)$. Comme $h$ est finie et continue en $\theta$, alors selon un argument déjà utilisé, les $h_{n}$ sont uniformément majorées sur un voisinage de $\theta$ et donc $h_{n}(\theta)$ est finie et $h_{n} \in \Gamma\left(\mathbb{R}^{q}\right)$ pour tout $n$ suffisamment grand. Ce qui achève la preuve.

Démonstration du théorème 5.5. $\left(\mathcal{P}_{n}\right)$ et $(\mathcal{P})$ admettent au moins une solution : En effet, $(\mathcal{P})$ admet au moins une solution car $\Phi(., \theta)$ est inf-compacte. D'autre part, les fonctions $\Phi_{n}(., \theta)$ sont convexes et sci. Leur épi-limite inférieure [1], lime $\Phi_{n}(., \theta)$ vérifie en vertu du lemme 5.3 la condition :

$$
\forall x \in X, \underline{\lim } e \Phi_{n}(x, \theta)=\inf \left\{\underline{\lim } \Phi_{n}\left(x_{n}, \theta\right) / x_{n} \stackrel{\|\cdot\|}{\longrightarrow} x\right\} \geq \Phi(x, \theta) .
$$

La fonction lime $\Phi_{n}(., \theta)$ étant sci, minorée par une fonction inf-compacte est inf-compacte. Les fonctions $\Phi_{n}(., \theta)$ sont donc uniformément inf-compactes d'après le lemme 5.6. Soit $\alpha \in \mathbb{R}$ tel que $\sup _{n \geq N} h_{n}(\theta)<\alpha$. Il existe pour tout $n \geq N, x_{n} \in X$ tel que $\Phi_{n}\left(x_{n}, \theta\right)<\alpha$. D'autre part, il existe $K_{\alpha}$ un compact non vide et $N^{\prime} \geq N$ tels que pour tout $n \geq N^{\prime}$, les ensembles $\left\{x / \Phi_{n}(x, \theta) \leq \alpha\right\}$ sont des convexes fermés non vides contenus dans $K_{\alpha}$. Les fonctions $\Phi_{n}(., \theta), n \geq N^{\prime}$ sont donc inf-compactes [41] et les problèmes $\left(\mathcal{P}_{n}\right)_{n \geq N^{\prime}}$ admettent au moins une solution. Le reste suit classiquement comme dans la preuve du théorème 5.2 , puisque les fonctions $\Phi_{n}(., \theta)$ sont uniformément inf-compactes et les fonctions marginales $h_{n}$ sont uniformément majorées sur un voisinage de $\theta$ avec $h_{n} \stackrel{e}{\longrightarrow} h$.

Enfin, comme application à la programmation mathématique, nous déduisons du théorème 5.5 , le corollaire 5.1 de [34] qui se démontre exactement de la même façon. Remarquons enfin que dans le théorème précédent, en général $\Phi_{n}(., \theta) \stackrel{e}{\rightarrow} \Phi(., \theta)$. Ainsi, la conclusion du théorème 5.5 ne peut pas être déduite du théorème 7.33 de $[42]$.

Remerciements. Les auteurs expriment leur reconnaissance au rapporteur de ce papier, pour ses remarques judicieuses et ses suggestions fructueuses qui ont contribué à l'amélioration de la rédaction finale de cet article. 


\section{RÉFÉRENCES}

[1] H. Attouch, Variational convergence for functions and operators, Applicable Mathematics Series. Pitman, London (1984).

[2] H. Attouch, D. Azé and R.J.-B. Wets, Convergence of convex-concave saddle functions; continuity properties of the LegendreFenchel transform with applications to convex programming and mechanics. Ann. Inst. Henri Poincaré 5 (1988) 537-572.

[3] H. Attouch and G. Beer, On the convergence of subdifferentials of convex functions. Arch. Math. 60 (1993) 389-400.

[4] H. Attouch and H. Brezis, Duality for the sum of convex functions in general Banach spaces. Publications AVAMAC, Perpignan, 84-10. Av (1984).

[5] H. Attouch and R.J.-B. Wets, Quantitative stability of variational systems: II. A framework for nonlinear conditionning. IIASA working paper (1988) 88-89.

[6] H. Attouch and R. J. -B. Wets, Quantitative stability of variational systems: I. The epigraphical distance. Trans. Amer. Math. Soc. 328 (1991) 695-729.

[7] D. Azé and J.-P. Penot, Operations on convergent families of sets and functions. Optimization 21 (1990) 521-534.

[8] B. Bank, J. Guddat, D. Klatte, B. Kummer and K. Tammer, Nonlinear parametric optimization. Akademie Verlag (1982).

[9] G. Beer, Topologies on closed and closed convex sets and the Effros measurability of set valued functions, in Sém. d'Anal. Convexe, Montpellier (1991), exposé No. 2, 2.1-2.44.

[10] G. Beer, The slice topology: A viable alternative to Mosco convergence in nonreflexive spaces. Nonlinear. Anal. Theo. Meth. Appl. 19 (1992) 271-290.

[11] G. Beer and J. Borwein, Mosco convergence and reflexivity. Proc. Amer. Math. Soc. 109 (1990) 427-436.

[12] G. Beer and R. Lucchetti, Convex optimization and the epi-distance topology. Trans. Amer. Math. Soc. 327 (1991) 795-813.

[13] G. Beer and R. Lucchetti, The epi-distance topology: Continuity and stability results with applications to convex optimization problems. Math. Oper. Res. 17 (1992) 715-726.

[14] G. Beer and R. Lucchetti, Weak topologies for the closed subsets of a metrizable space. Trans. Amer. Math. Soc. 335 (1993) 805-822.

[15] N. Bourbaki, Espaces vectoriels topologiques. Masson, Paris (1981).

[16] H. Brezis, Analyse fonctionnelle, théorie et applications. Masson, Paris (1983).

[17] C. Castaing and M. Valadier, Convex analysis and measurable multifunctions. Lect. Notes Math. 580 (1977).

[18] J. Dieudonné, Sur la séparation des ensembles convexes. Math. Annal. 163 (1966) 1-3.

[19] S. Dolecki, G. Salinetti and R.J.-B. Wets, Convergence of functions: equi-semicontinuity. Trans. Amer. Math. Soc. 276 (1983) 409-429.

[20] A.L. Dontchev and T. Zolezzi, Well-posed optimization problems. Lect. Notes Math. 1543 (1993).

[21] I. Ekeland et R. Temam, Analyse convexe et problèmes variationnels. Dunod, Paris (1974).

[22] K. El Hajioui, Convergences variationnelles: approximations inf-convolutives généralisées, stabilité et optimisation dans les espaces non réflexifs. Thèse de Doctorat, Université Ibn Tofail, Kénitra (2002).

[23] K. El Hajioui et D. Mentagui, Sur la stabilité d'une convergence variationnelle dans les espaces de Banach généraux, en préparation.

[24] J. Hadamard, Sur les problèmes aux dérivées partielles et leur signification physique. Publ. Univ. Princeton 13 (1902) $49-52$.

[25] J. Hadamard, Lectures on Cauchy's problem in linear partial differential equations. Dover (1953).

[26] J.L. Joly, Une famille de topologies et de convergences sur l'ensemble des fonctionnelles convexes. Thèse Grenoble (1970).

[27] G. Köthe, Topological vector spaces (I, II). Springer (1969, 1979).

[28] J. Lahrache, Stabilité et convergences dans les espaces non réflexifs, in Sém. d'Anal. Convexe Montpellier, exposé No. 10 (1991).

[29] P.J. Laurent, Approximation et optimisation. Hermann, Paris (1972).

[30] L. Mclinden and R.C. Bergstrom, Preservation of convergence of convex sets and functions in finite dimensions. Trans. Amer. Math. Soc. 286 (1981) 127-142.

[31] D. Mentagui, Inf-convolution polaire, stabilité de l'épi-convergence et estimation de la rapidité de convergence d'une suite de compacts. Thèse Rabat (1988).

[32] D. Mentagui, Problèmes d'optimisation biens posés et convergences variationnelles. Théorie et applications dans le cadre de l'optimisation non différentiable. Thèse d'État, F.U.N.D.P., Namur (1996).

[33] D. Mentagui, Caractérisation de la stabilité d'un problème de minimisation associé à une fonction de perturbation particulière. Pub. Inst. Math. 60 (1996) 65-74.

[34] D. Mentagui, Analyse de récession et résultats de stabilité d'une convergence variationnelle. Application à la théorie de la dualité en programmation mathématique. ESAIM: COCV 9 (2003) 297-315.

[35] D. Mentagui et K. El Hajioui, Convergences des fonctions convexes et approximations inf-convolutives généralisées. Publ. Inst. Math., Nouvelle série 86 (2002) 123-136.

[36] J.J. Moreau, Fonctionnelles convexes. Sém. sur les E.D.P. collège de France, Paris (1967).

[37] U. Mosco, Approximation of the solutions of some variational inequalities. Ann. Scuola Normale Sup. Pisa 21 (1967) 373-394.

[38] U. Mosco, On the continuity of the Young-Fenchel transform. J. Math. Anal. Appl. 25 (1971) 518-535.

[39] R. Phelps, Convex functions, monotone operators and differentiability. Lect. Notes Math. 1364 (1989). 
[40] H. Radström, An imbedding theorem for spaces of convex sets. Proc. Amer. Math. Soc. 3 (1952) 165-169.

[41] R.T. Rockafellar, Convex Analysis. Princeton Univ. Press (1970).

[42] R.T. Rockafellar and R.J.-B. Wets, Variational analysis. Springer (1998).

[43] Y. Sonntag and C. Zalinescu, Set convergences: An attempt of classification, in Proc. of Intl. Conf. on Diff. Equations and Control theory, Iasi, Romania, August (1990) 199-226.

[44] A.N. Tikhonov, Stability of inverse problems. Dokl. Akad. Nauk. USSR 39 (1943) 176-179.

[45] A.N. Tikhonov, Solution of incorrectly formulated problems and the regularization methods. Soviet Math. Dokl. 4 (1963) 1035-1038.

[46] A.N. Tikhonov, Methods for the regularization of optimal control problems. Soviet Math. Dokl. 6 (1965) 761-763.

[47] A.N. Tikhonov and V. Arsenine, Methods for solving ill-posed problems. Nauka (1986).

[48] R.J.-B. Wets, A formula for the level sets of epi-limits and some applications, Mathematical theories of optimization, J.P. Cecconi and T. Zolezzi Eds., Lect. Notes Math. 983 (1983).

[49] R.A. Wijsman, Convergence of sequences of convex sets, cones and functions. Bull. Amer. Math. Soc. 70 (1964) $186-188$.

[50] R.A. Wijsman, Convergence of sequences of convex sets, cones and functions II. Trans. Amer. Math. Soc. 123 (1966) 32-45.

[51] T. Zolezzi, On stability in mathematical programming. Math. Programming 21 (1984) 227-242.

[52] T. Zolezzi, Continuity of generalized gradients and multipliers under perturbations. Math. Oper. Res. 10 (1985) 664-673.

[53] T. Zolezzi, Stability analysis in optimization. Lect. Notes Math. 1990 (1986) 397-419. 